\title{
Development of a simplified method for optimally sizing hot water storage tanks subject to short-term intermittent charge/discharge cycles
}

by

\author{
Danisz Stalinski
}

A thesis submitted to the Faculty of Graduate and Postdoctoral Affairs in partial fulfillment of the requirements for the degree of

Master of Applied Science

in

Sustainable Energy

Carleton University

Ottawa, Ontario

(C) 2020

Danisz Stalinski 


\section{Abstract}

Hot water storage tanks play a pivotal role in the transition to low carbon energy systems by increasing energy efficiency and allowing a range of non-conventional intermittent energy sources in the energy mix such as renewable energy and recovered waste heat. Apart from lowering GHG emissions, sizing these systems appropriately can lead to significant reductions in annual energy cost. In the current study, a simple analytical method is developed to optimally size hot water storage tanks using only the system residual heating profile and the following system characteristics: the heat source temperature, the load service temperature, the auxiliary energy price, and the tank's surrounding environmental temperature. A transient numerical model (TRNSYS) of a hot water storage tank system is developed to simulate randomly generated residual heating profiles under 36 distinct scenarios. For each residual heating profile, the optimal storage tank volume is determined by coupling the TRNSYS model to an optimization framework developed in Matlab®. Fast Fourier transform techniques are used to decompose each residual heating profile, and ordinary least-squares regression models are used to relate the residual heating profile's component amplitudes and periods to the optimal storage volume. Results show that all scenarios with a source temperature of $95^{\circ} \mathrm{C}$, a source to load temperature difference of at least $35{ }^{\circ} \mathrm{C}$, and an auxiliary energy price of at least $0.105 \mathrm{USD} / \mathrm{kWh}$ have associated $\mathrm{R}^{2}$ values of 0.8 or greater, indicating the proposed sizing method is able to predict the optimal storage tank volume with a high degree of confidence. A case study is presented to demonstrate the proposed sizing method for a hypothetical solar-thermal DHW system in Montréal, Canada. 


\section{Acknowledgements}

A good tour guide is one who allows the tourist enough time to stop and smell the flowers, while still loosely following the tour's schedule. If the guide rushes the tourist to the last destination, the tourist feels cheated, having experienced little on the trip. On the other hand, a tour guide who allows the tourist to wander aimlessly prevents him from ever reaching the main attractions. Over the past two years or so I have been a tourist exploring the country of graduate studies, and I would like to thank my supervisor, Dr. Jean Duquette, for being a good tour guide. In times of creativity he allowed me the freedom to explore new ideas; in times of chaos he guided me back onto the path to completion.

Of course, the success of any trip is influenced by those with whom you embark, so I am thankful that I had a great group of travel companions. I want to thank my parents, Tadeusz and Katarzyna, my girlfriend Danielle, and my good friend Steven for their persistent support. I also met a few memorable characters along the way, most notably Joseph, with whom I've shared countless laughs.

Thank you to the Natural Sciences and Engineering Research Council for providing financial support for this project through a Canada Graduate Scholarship (Master's). 


\section{Table of Contents}

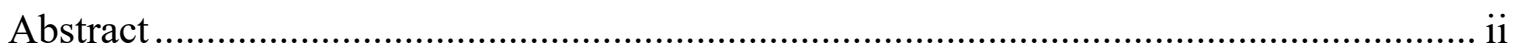

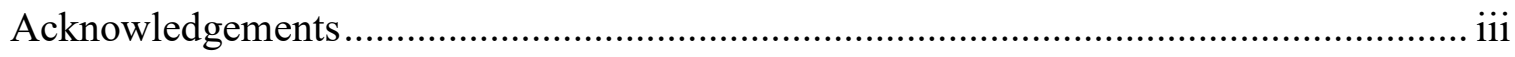

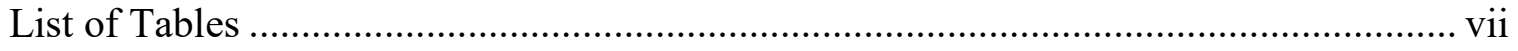

List of Figures ................................................................................................ viii

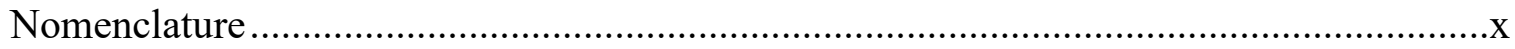

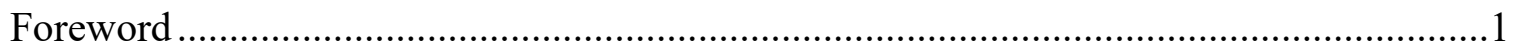

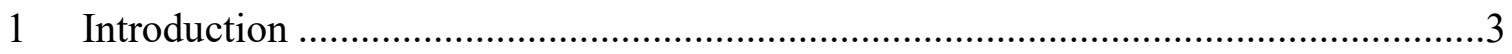

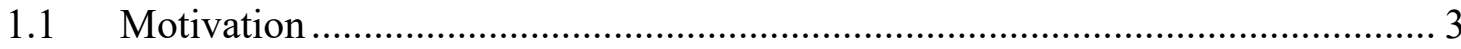

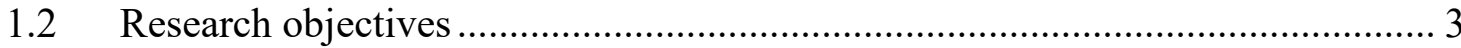

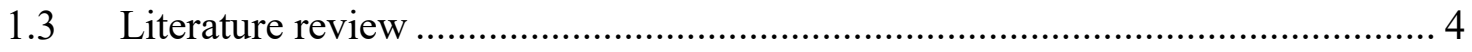

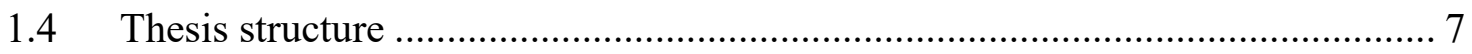

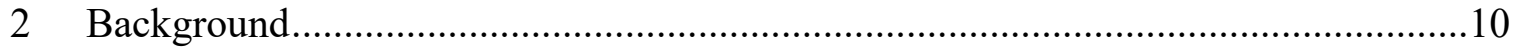

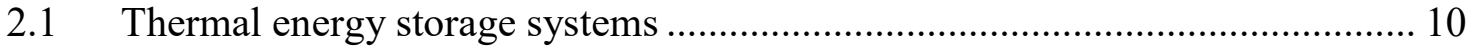

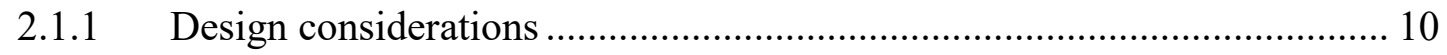

2.1.2 Thermal energy storage alternatives ................................................... 12

2.1.3 Numerical modelling of hot water storage tanks .................................. 13

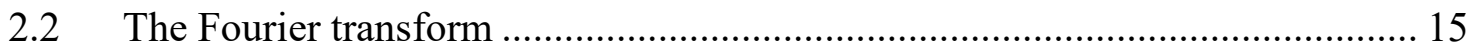

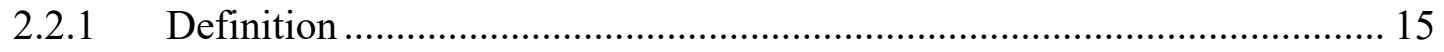




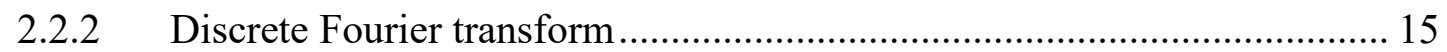

2.2.3 Fast Fourier transform....................................................................... 16

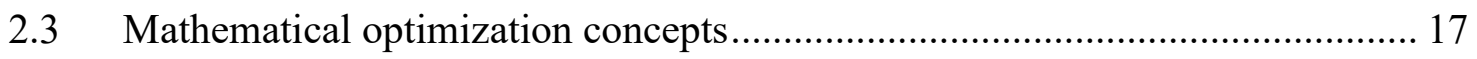

2.3.1 Optimization problems and dimensionality ............................................ 17

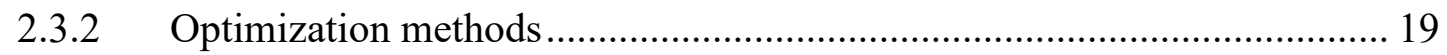

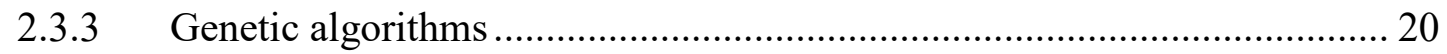

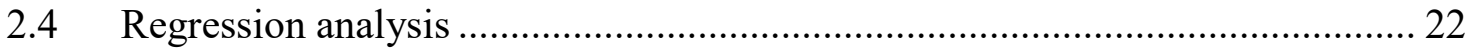

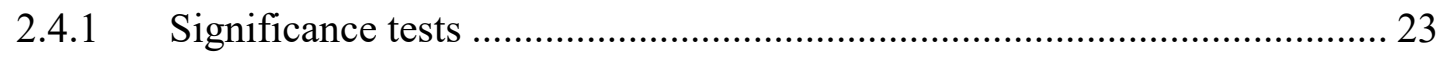

2.4.2 Other modelling options .................................................................... 24

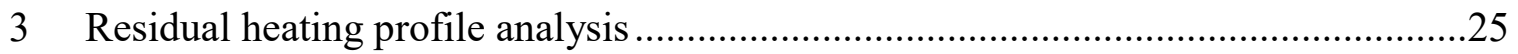

3.1 Relationship between residual heating profile and hot water storage volume ... 26

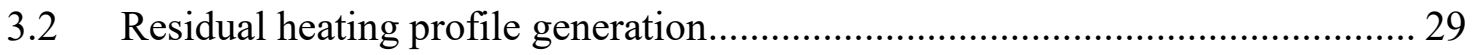

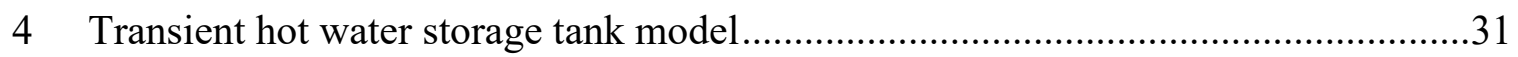

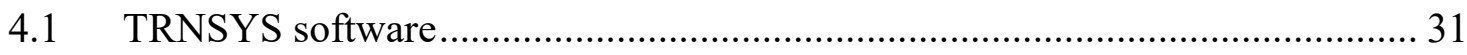

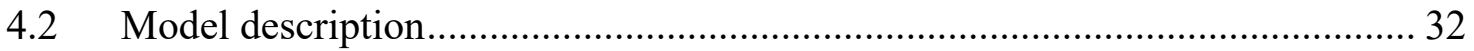

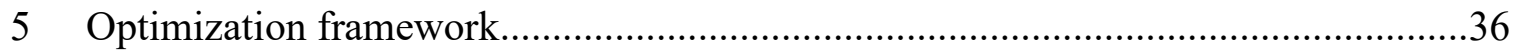

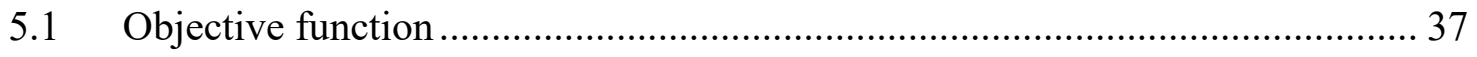

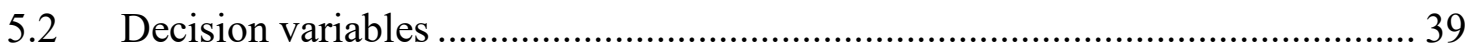

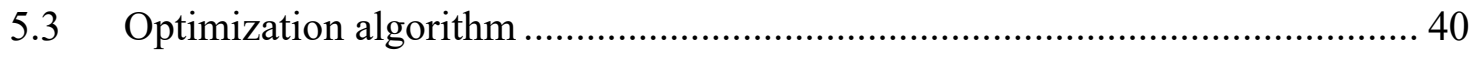

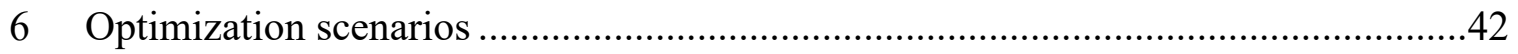


7 Development of a generalized regression model.................................................44

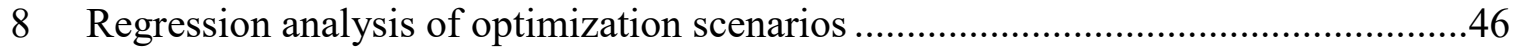

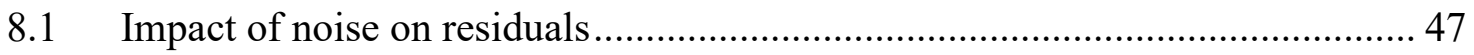

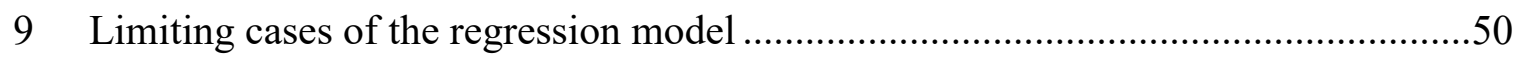

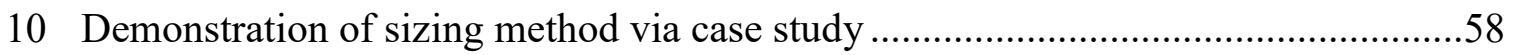

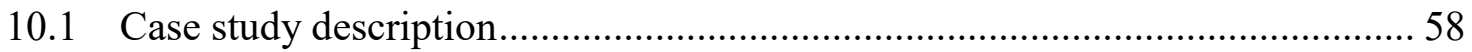

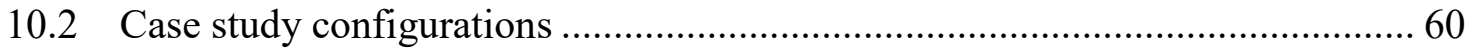

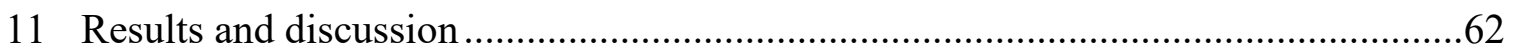

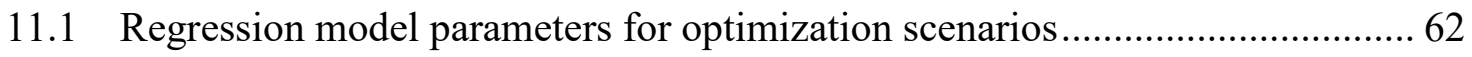

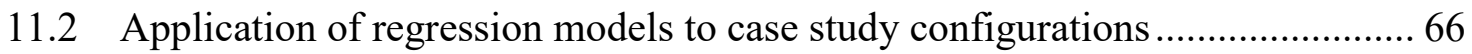

11.3 Sizing method applicability and unresolved issues.................................... 69

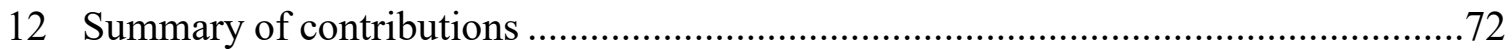

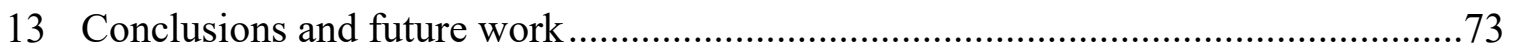

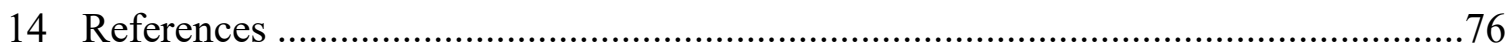




\section{List of Tables}

Table 2.1: Important considerations for TES systems by storage parameter, adapted from Bradford [14]. 11

Table 5.1: Component fixed unit costs used to quantify the objective function 39 Table 5.2: Fixed values corresponding to each storage tank variable considered in current study. 40

Table 6.1: Values of extraneous variables used to develop optimization scenarios, and corresponding selection rationale 43

Table 9.1: Index for selecting the value of $k p$ for a residual with two sinusoids. 55

Table 10.1: Case study configurations based on varying combinations of extraneous parameters 61

Table 11.1: Regression model parameters $(c 1-c 5)$ and corresponding $R 2$ value as a function of hot water storage tank source and load temperatures, tank environment, and auxiliary energy price for 36 optimization scenarios 64

Table 11.2: Case study results by configuration. Vopt, and Vopt represent the simulated and predicted optimal hot water storage tank volume, respectively; whereas erel, and NPC represent the relative model error and system net present cost, respectively.................. 66 Table 11.3: Parameters used for calculating Vopt for case study configuration B 


\section{List of Figures}

Figure 2.1: Flow diagram depicting the general steps of a typical genetic algorithm optimization method. 22

Figure 3.1: Normalized source and load profiles corresponding to solar thermal [20], and CHP [21]; and DHW [22], and SH [23] systems, respectively, over a 24-hour period on May $10^{\text {th }}$. The profiles are arbitrarily selected from datasets taken over several years.... 26 Figure 3.2: Two hypothetical residual heating profiles. Sinusoidal profiles can be characterized using their characteristic amplitude, period and phase. 27

Figure 3.3: A hypothetical residual heating profile composed of two sinusoids (and noise) is shown in (a). Using the FFT algorithm, the profile is decomposed into the two underlying sinusoids; the amplitudes and phases are plotted against frequency in (b) and (c), respectively. 28

Figure 4.1: Schematic of transient numerical model developed [24] to assess the performance of a hot water storage tank of any given volume as a function of residual heating profile. 33

Figure 5.1: Optimization framework used to determine optimal hot water storage tank volume for any given residual heating profile. 36

Figure 8.1: Probability distributions of the relative model error for a given scenario subject to varying noise levels during both model fitting (blue) and testing (orange). Bias is introduced into the model in both (a) where the model is fit to noiseless data, and (b) where the model is fit to data where $\sigma r e l=0.2$.

Figure 9.1: Optimal storage size plotted as a function of sinusoid amplitude and period for residuals with $n=1$ 
Figure 9.2: Relative model error as a function of $h$, $\phi r e l$ and arel. Plots (a) and (b) represent sample relative model errors for cases with $h=2$ and $h=3$ respectively........... 54

Figure 9.3: Probability distributions of relative model error for sinusoids with $n=2$. The blue distribution represents the relative model errors when harmonics are ignored (using Eq. (23)), while the orange distribution represents the relative model errors when additional model parameters are used to reduce the overprediction bias (using Eq. (30)) ............... 57

Figure 10.1: Schematic of transient numerical model developed to demonstrate the proposed sizing method on a case study solar thermal domestic hot water system located

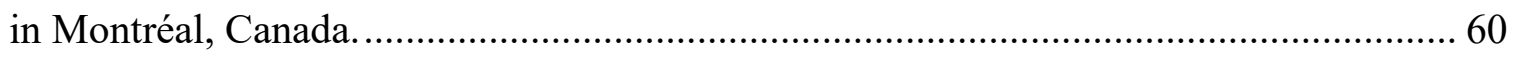

Figure 11.1: Box-and-whisker plots depicting relative model error, erel, probability distributions for 36 optimization scenarios. (a), (b), (c), and (d) refer to scenarios with source and load temperatures of $95^{\circ} \mathrm{C}$ and $60^{\circ} \mathrm{C}, 95^{\circ} \mathrm{C}$ and $40^{\circ} \mathrm{C}, 75^{\circ} \mathrm{C}$ and $60^{\circ} \mathrm{C}$, and $75^{\circ} \mathrm{C}$ and $40^{\circ} \mathrm{C}$, respectively 65

Figure 11.2: Annual residual heating profile (a), and corresponding periodogram (b) for case study configuration $\mathrm{B}$. 67

Figure 11.3: Objective function for case study configuration B showing values of Vopt, and Vopt nearly overlapping. 69 


\section{Nomenclature}

\section{Symbols}

\begin{tabular}{|c|c|}
\hline$a$ & amplitude $(\mathrm{kW})$ \\
\hline$b_{k}$ & scaling factor for harmonic of type $k$ \\
\hline$C_{\text {aux }}$ & cost of the auxiliary heater (USD) \\
\hline$C_{c a p}$ & capital cost of storage tank (USD) \\
\hline$C_{\text {cool }}$ & cost of the cooling tower (USD) \\
\hline$C_{\text {energy }}$ & costs of energy use (USD) \\
\hline$C_{\text {energy,a }}$ & annual energy cost (USD) \\
\hline$c_{i}$ & regression model parameter for sinusoid $i\left(\mathrm{~m}^{3} / \mathrm{kWh}\right)$ \\
\hline$C_{\text {ins }}$ & cost of insulation (USD) \\
\hline$C_{\text {maint }}$ & cost of maintenance (USD) \\
\hline$C_{p}$ & specific heat capacity $(\mathrm{J} / \mathrm{kgK})$ \\
\hline$E_{\text {annual }}$ & annual energy use (kWh) \\
\hline$e_{\text {rel }}$ & relative model error \\
\hline$f$ & frequency $\left(\mathrm{hr}^{-1}\right)$ \\
\hline$F(t)$ & a real-valued function input into the Fourier transform \\
\hline$h$ & natural number multiple relating two harmonic periods \\
\hline$i$ & discount rate \\
\hline$k$ & identifier for different types of harmonics \\
\hline$k_{p}$ & the harmonic identifier applicable to two sinusoids \\
\hline
\end{tabular}




\begin{tabular}{|c|c|}
\hline$\dot{m}_{k}$ & mass flow rate at point $\mathrm{k}(\mathrm{kg} / \mathrm{s})$ \\
\hline$M F$ & maintenance factor \\
\hline$n$ & number of sinusoids \\
\hline$p$ & period (hr) \\
\hline$Q$ & heat $(\mathrm{kWh})$ \\
\hline$Q_{a u x}(t)$ & heat transfer rate at the auxiliary heater $(\mathrm{kW})$ \\
\hline$Q_{\text {cool }}(t)$ & heat transfer rate at the cooling tower $(\mathrm{kW})$ \\
\hline$Q_{l o a d}(t)$ & heat transfer rate at the load $(\mathrm{kW})$ \\
\hline$Q_{\text {source }}(t)$ & heat transfer rate at the source $(\mathrm{kW})$ \\
\hline$R^{2}$ & coefficient of determination \\
\hline$r_{E}$ & auxiliary energy cost (USD/kWh) \\
\hline$r(t)$ & residual heating profile $(\mathrm{kW})$ \\
\hline$S S_{\text {res }}$ & sum of squared residuals \\
\hline$S S_{\text {tot }}$ & total sum of squares \\
\hline$t$ & time $(\mathrm{hr})$ \\
\hline$T_{e n v}(t)$ & temperature of the tank environment $\left({ }^{\circ} \mathrm{C}\right)$ \\
\hline$T_{j}$ & temperature at point $\mathrm{j}\left({ }^{\circ} \mathrm{C}\right)$ \\
\hline$T_{\text {tank,bot }}(t)$ & bottom tank temperature $\left({ }^{\circ} \mathrm{C}\right)$ \\
\hline$T_{\text {tank,top }}(t)$ & top tank temperature $\left({ }^{\circ} \mathrm{C}\right)$ \\
\hline$V_{\text {opt }}$ & optimal volume $\left(\mathrm{m}^{3}\right)$ \\
\hline$\widehat{V}_{o p t}$ & predicted optimal volume $\left(\mathrm{m}^{3}\right)$ \\
\hline $\bar{V}_{o p t}$ & mean optimum storage volume $\left(\mathrm{m}^{3}\right)$ \\
\hline
\end{tabular}




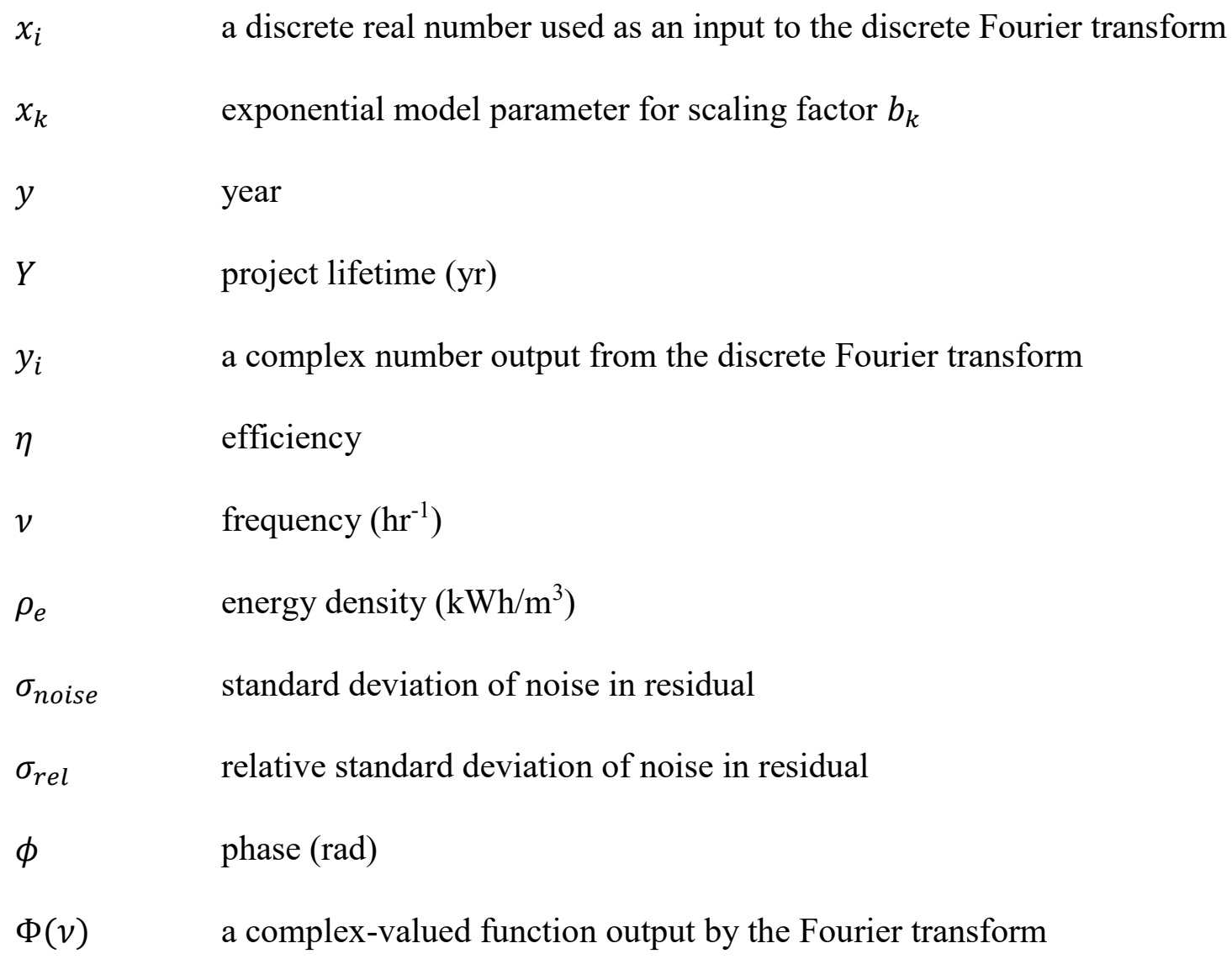

\section{Acronyms and Abbreviations}

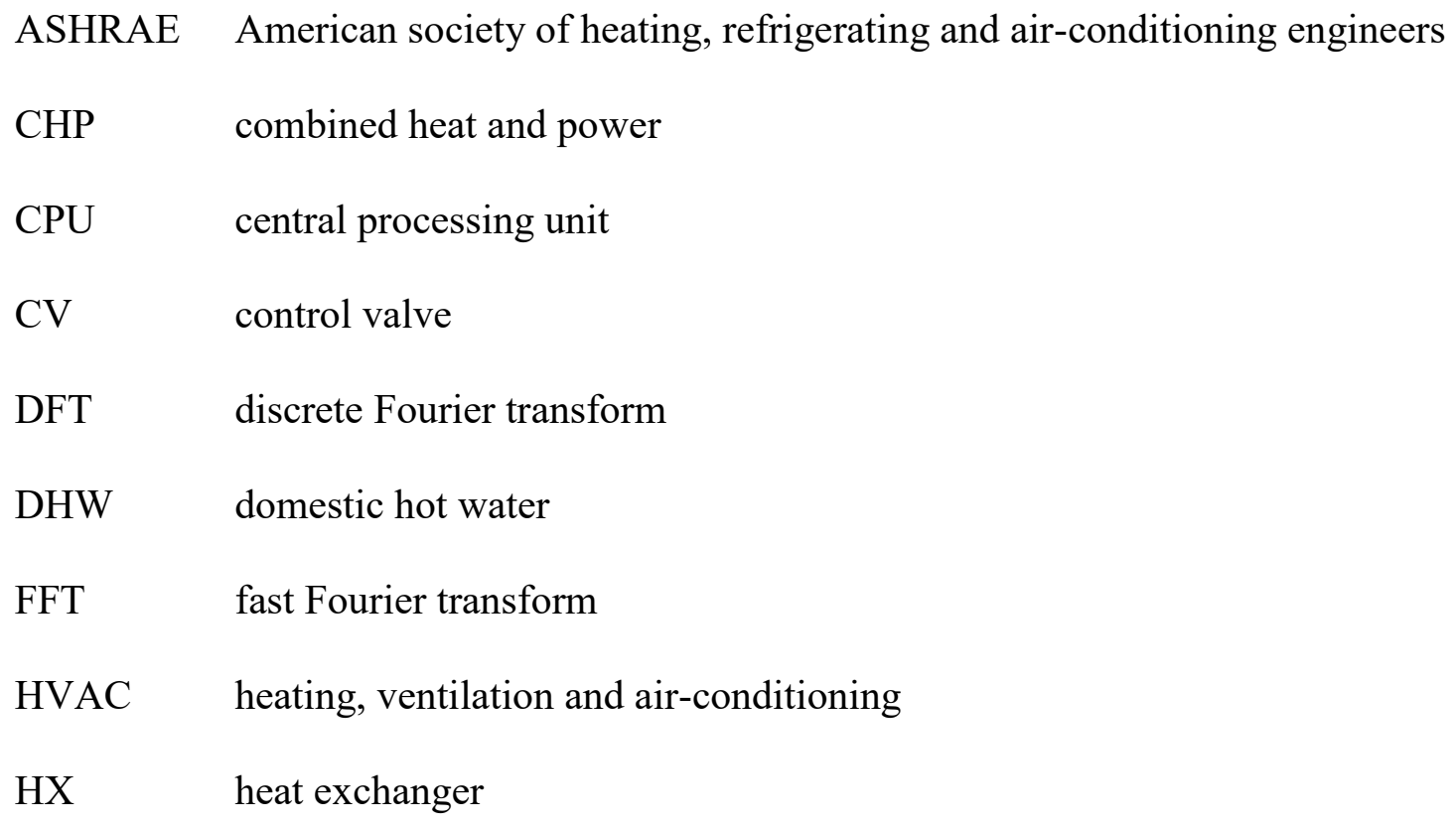




$\begin{array}{ll}\text { MLE } & \text { maximum likelihood estimator } \\ \text { MV } & \text { mixing valve } \\ \text { NPC } & \text { net present cost } \\ \text { OLS } & \text { ordinary least squares } \\ \text { RAM } & \text { random access memory } \\ \text { RSM } & \text { response surface model } \\ \text { SH } & \text { space heating } \\ \text { TES } & \text { thermal energy storage } \\ \text { VSP } & \text { variable speed pump }\end{array}$

\section{Subscripts}

$\begin{array}{ll}\text { aux } & \text { auxiliary heater } \\ \text { cool } & \text { cooling tower } \\ \text { E, e } & \text { energy } \\ \text { enrg } & \text { relating to energy consumption } \\ \text { enrg,a } & \text { relating to annual energy consumption } \\ \text { env } & \text { environmental } \\ i, j, k & \text { index variables } \\ \text { ins } & \text { insulation of the hot water storage tank } \\ \text { load } & \text { the system heat load } \\ \text { maint } & \text { maintenance of the hot water storage tank } \\ \text { noise } & \text { relating to the noise applied to the residual profile } \\ \text { opt } & \text { optimal }\end{array}$


$\mathrm{p}$

rel

res

source

tank,bot

tank,top

tot parameter

relative

residual

the intermittent heat source

the bottom of the hot water storage tank

the top of the hot water storage tank

total 


\section{Foreword}

During my undergraduate studies I was fortunate enough to spend some time on a work placement with a company that owns and operates renewable energy technologies. In this work placement I became fascinated with the energy sector and developed a drive to further explore sustainable energy alternatives. This drive led me to pursue a master's degree in the sustainable energy engineering and policy master's program at Carleton, where my first assignment was to select a suitable research topic for this thesis. Thinking back to my work experience, I recalled several instances where I had tried to develop some improved operation strategies, refine some forecast tool, or implement some more efficient algorithm. I often found myself searching the literature for ideas, only to face considerable difficulty in applying what I found to my own work. While this can be partly attributed to my own lack of experience or understanding, I genuinely think that there is a gap in the literature for simple methods or techniques that can be readily implemented by engineers. For this reason, my desire above all else was to work on a project that while still novel enough to contribute to the literature, was also practical enough to potentially be directly used by an engineer.

This thesis presents a sizing methodology for hot water storage tanks that can be utilized to increase the efficiency of some thermal energy systems. By design, the application of this method does not require extensive amounts of expertise, time or other resources. I hope that it may prove useful to engineers and practitioners, or that it may be further refined by others until it is.

The work completed in this thesis has been submitted to the international peer reviewed journal Applied Energy (see reference below) and is currently under review. 
- D. Stalinski and J. Duquette, "Development of a simplified method for optimally sizing hot water storage tanks subject to short-term intermittent charge/discharge cycles," Applied Energy [under review]. 


\section{Introduction}

\subsection{Motivation}

Space and domestic hot water heating demands currently account for roughly $80 \%$ and $63 \%$ of the total energy use by end use in the Canadian residential and commercial building sectors, respectively [1]. Traditionally, these heating demands have largely been met using on-demand fossil fuel based heating systems [1]. However, recently adopted energy efficiency standards, codes, and practices in the building sector are motivating building owners and developers to move away from fossil fuel based energy sources towards lower carbon alternatives such as renewable energy generation and waste heat recovery [2], [3]. Although these alternatives are abundant and readily available, their intermittent nature presents a significant barrier to their widespread integration into our existing energy system. Temporal variations between sources and loads create conditions where energy cannot always be utilized when it is available. To remedy this situation, a thermal buffer such as a hot water storage tank can be used, however, proper care must be taken to size the system appropriately. Under-sizing hot water storage tanks can lead to unmet performance criteria and higher usage of potentially costly back-up energy sources, whereas over-sizing can lead to undesirable performance and poor system costeffectiveness due to associated high initial capital investments [4].

\subsection{Research objectives}

The objective of this study is to develop a simplified analytical method built upon both mathematical programming and simulation models that can be used for optimally sizing 
hot water storage tanks subject to both temporally varying heating sources and loads. The only assumption made regarding the nature of the source and load is that any variations occur on a relatively short time scale (i.e. periods not exceeding 48 hours) which makes this method suitable for diurnal storage, but not for seasonal storage. Fortunately, many common sources and loads (e.g. space and domestic hot water heating loads, and solar and wind sources) exhibit such short-term variations, making this method applicable to a wide range of conventional systems. Additionally, the method does not require the use of specific software or prohibitive amounts of input data. In fact, the only required inputs are the heating source and load time series (the difference of which make up the residual heating profile), the heating source and load temperatures, the climate zone in which the system is to be installed, and the auxiliary energy price. Therefore, with only limited information, a system designer may quickly obtain an accurate estimate for the optimal storage tank volume. To the authors' knowledge, no such method has been developed for sizing hot water storage tanks.

\subsection{Literature review}

Several methods have been proposed in the literature for optimally sizing hot water storage tanks. These methods can be grouped into the following three distinct categories: analytical methods, mathematical programming methods, and simulation-based methods [5]. The first category of methods (i.e. analytical methods) makes use of models or algorithms to directly determine the optimal storage tank volume and can vary widely with regards to level of complexity. Pinnau and Breitkopf [6] used an analytical method to size multiple hot water storage tanks for an apartment building located in Potsdam, Germany. Their method involves decomposing the hot water heating residual profile (i.e. the difference 
between heating energy input from a dispatchable boiler and output heating demand) into a set of cosine functions using a fast Fourier transform (FFT). The dominant frequencies (i.e. those with the largest amplitudes) are selected from this set, and each is individually assigned to a unique hot water tank. Thus, each tank is responsible for storing according to a single dominant frequency, and throughout operation continuously repeats cycles of charging to capacity and discharging to depletion. Implementation of their method had the effect of flattening the load duration curve of the heat generator which in turn improved overall performance. In a separate study, Bianchi et al. [7] developed an analytical method for sizing hot water storage capacity for residential buildings based on the load profile for a combined heat and power (CHP) system, however rather than using frequency analysis they developed a set of charts to relate the optimal storage volume directly to the peak heating load and CHP unit output. Aside from the analytical tank sizing methods found in the literature, even simpler design-day spreadsheet methods can be obtained from engineering reference handbooks such as the American Society of Heating, Refrigerating, and Air-Conditioning Engineers (ASHRAE) handbook on heating, ventilation, and airconditioning (HVAC) equipment [8].

The second category of methods (i.e. mathematical programming methods) abstracts the problem of energy storage sizing into a set of variables and equations that are formulated as an optimization problem. A solver is used to determine parameter values that optimize the value of the objective function, while satisfying any constraints in the problem. For example, Kavvadias et al. [9] examined the effect of including a hot water storage tank in a trigeneration plant operating under different operating strategies using a mixed integer programming model. Their findings demonstrated that the inclusion of the 
storage tank increased project profits, with the increase becoming more significant as the operating hours of the boiler and CHP unit were reduced. Cabello et al. [10] compared the performance of two mathematical programming solvers against that of a genetic algorithm based simulation approach for sizing a hot water storage tank and solar collectors for a solar thermal energy plant. Ultimately, they argue in favour of the genetic algorithm-based approach, due to its reduced susceptibility of getting "stuck" in local optima.

The third category of methods (i.e. simulation-based methods) involve the emulation of temporally dependent physical processes associated to a given system of interest in a numerical environment. From initial input data and numerical representations of the system components, simulations are conducted over a predefined time horizon to examine how the system responds in time. Model outputs are collected throughout the simulation, and typically many simulations are conducted over various system configurations to determine optimal performance. In their study of dispatchable biomass boiler systems, Wang et al. [11] simulated building energy systems with different hot water storage tank volumes to determine the optimal volume for a variety of boiler capacities and load profiles. They found a linear relationship between the optimal tank volume and boiler capacity, and ultimately recommend sizing the boiler capacity to roughly twice the average annual heating demand. By distilling a wide range of simulation results into a numerical correlation, they also provided an analytical framework that can be more easily applied by other designers for sizing similar components. Rogríguez-Hidalgo et al. [12] developed their own transient simulation tool to study the co-optimization of solar collector area and hot water tank storage volume for solar thermal heating systems. They found that both 
energy savings and annual solar fraction are maximized at tank volume to collector area ratios of approximately $0.09 \mathrm{~m}$, beyond which no additional gains are achieved.

Of the three categories mentioned above, analytical methods and simplified simulation environments are generally the most attractive to designers due to their ease of implementation, but their simplicity generally precludes them from finding solutions that are truly optimal. Some analytical methods are developed as a generalization of more specific simulation-based and mathematical programming methods; however, these are typically developed in a specific context (e.g. the simulation-analytical method presented by Wang et al. [11] is developed assuming a dispatchable source, for five different load profiles), which may not always match the circumstances of the potential user. On the other hand, both mathematical programming and simulation-based methods are ideal for optimally sizing hot water storage tanks because they can be custom-tailored to the designer's system. Unfortunately, this benefit also presents a challenge, because custombuilding new models on a case-by-case basis requires considerable effort and advanced numerical analysis tools (e.g. specialized mathematical optimization, and modeling software). These tools often come with a steep learning curve that may be prohibitive for designers who lack the time or skill to implement them. Moreover, many of these tools come with a significant up-front cost, which makes them impractical for some users.

\subsection{Thesis structure}

Before delving into the development of the sizing method, Chapter 2 provides an overview of general background information that may be useful for understanding the subsequent sections. The proposed sizing method is based on a response surface model (RSM) approach [13] and comprises the following five steps: 
1. Determine a suitable set of predictor variables. In the current work, predictor variables are obtained by implementing a FFT waveform conversion algorithm on a randomly generated set of residual heating profiles. The methods used for generating these profiles and corresponding predictor variables are described in Chapter 3.

2. Develop a transient simulation model of a hot water storage tank to assess performance as a function of residual heating profile. The methods used to develop the model are described in Chapter 4.

3. Integrate the simulation model within an optimization framework to determine the optimal storage volume for any residual heating profile. Details regarding the optimization framework are provided in Chapter 5.

4. Identify multiple optimization scenarios. Each scenario represents a unique combination of extraneous variables that have an influence on the optimal hot water storage tank volume (e.g. auxiliary energy price, tank environment, and heating source and load temperatures). The rationale used for selecting these variables and their corresponding values are described in Chapter 6.

5. For each optimization scenario, perform regression analysis to establish a relationship (i.e. a response surface) between the optimal hot water storage tank volume and the stated predictor variables. The steps used to develop this relationship are provided in Chapters 7 through 8, with Chapter 9 serving to discuss a limiting case of the relationship.

The implementation and accuracy of the proposed sizing method is demonstrated through a case study in which a hot water storage tank is sized to meet the domestic hot water 
(DHW) needs of a large residential building located in Montréal, Canada. The details of this case study are presented in Chapter 10.

The results of the regression analysis, as well as the results of applying the regression models to the case study, are discussed in Chapter 11. The thesis closes with a short comment on the contributions of this work to the broader literature in Chapter 12, followed by a summary of the main conclusions and recommendations for future research, which are provided in Chapter 13. 


\section{Background}

This section introduces some concepts that are important for the understanding of the research work. It is not meant to be an exhaustive or in-depth overview of relevant topics; rather the aim is to provide the reader with context of the terminology and concepts that are present in the thesis.

\subsection{Thermal energy storage systems}

\subsubsection{Design considerations}

The fundamental purpose of a thermal energy storage (TES) system is to balance temporally varying thermal sources and loads. The volume of the storage system is critical as it dictates the quantity of thermal energy that can be stored for later use. As was mentioned in Chapter 1.1, both under and over-sizing can lead to sub-optimal system performance. There are, however, several other important parameters that should be considered when designing a TES system. Table 2.1 below presents a list of these important parameters, which mainly influence the type of TES that is used in a system; different TES alternatives are described in the next section. 
Table 2.1: Important considerations for TES systems by storage parameter, adapted from Bradford [14].

\begin{tabular}{|c|c|}
\hline Storage parameter & Impact on a TES system \\
\hline energy density & $\begin{array}{l}\text { Increasing energy density allows one to store the same } \\
\text { amount of energy in a smaller volume, which reduces space } \\
\text { requirements that may be limited. }\end{array}$ \\
\hline power & $\begin{array}{l}\text { Aside from storing enough energy, a TES device should be } \\
\text { able to provide the energy quickly enough to meet the system } \\
\text { load. This is a property of the auxiliary components that move } \\
\text { thermal energy to and from the TES device. }\end{array}$ \\
\hline losses & $\begin{array}{l}\text { Different TES systems have different heat loss } \\
\text { characteristics, which affect the amount of energy that can be } \\
\text { stored, or equivalently the amount of time that a certain } \\
\text { amount of energy can be stored before being lost. }\end{array}$ \\
\hline lifetime & $\begin{array}{l}\text { TES projects are initially capital-intensive and aim to provide } \\
\text { a return on investment in the long term through energy } \\
\text { savings. TES devices with longer lifetimes are more likely to } \\
\text { be economically feasible }\end{array}$ \\
\hline $\begin{array}{l}\text { material availability } \\
\text { and cost }\end{array}$ & $\begin{array}{l}\text { The cost of the storage material used in a TES system directly } \\
\text { influence the project's economic feasibility. On the other } \\
\text { hand, the material's availability influences how likely the } \\
\text { project is to face delays in implementation. }\end{array}$ \\
\hline material safety & $\begin{array}{l}\text { Safety is of paramount importance in any engineering project, } \\
\text { so a TES device that does not require hazardous substances } \\
\text { will be preferred over one that does. }\end{array}$ \\
\hline geometry & $\begin{array}{l}\text { The shape of a TES device can influence heat transfer } \\
\text { processes as well as the stratification of sensible storage. }\end{array}$ \\
\hline
\end{tabular}




\subsubsection{Thermal energy storage alternatives}

Several types of TES technologies have been developed, each with their own benefits and challenges. The three main categories of TES are latent, thermochemical, and sensible [15]. Latent TES systems store and release thermal energy through a phase change process of a material, which generally results in higher energy densities than in sensible storage. The choice of storage material in a latent TES system is critical, as the material dictates the operating temperature of the TES device through its constant phase change temperature [4]. Examples of latent storage materials include ice/water, molten salts and paraffin waxes.

Thermochemical storage can refer to either a chemical reaction like the hydration of magnesium sulphate, or to a physical-chemical process like the adsorption of a substance (often water) onto another [15]. In either case, the thermochemical process is reversible through the addition or release of heat. Thermochemical TES methods often provide the highest energy densities of all TES methods; however, they are currently less developed than sensible and latent methods and can often be expensive.

Sensible TES systems store thermal energy through the temperature change of a storage medium. Examples of sensible storage include hot water tanks, large concrete blocks, aquifers, and solar ponds [4]. Materials that are inexpensive and have a high heat capacity are good candidates for use in sensible TES systems. Sensible hot water storage is one of the most well developed and practical types of TES. It relies on readily available and inexpensive water, does not involve any hazardous storage materials, and can provide long lifetimes due to easy maintenance. The disadvantage of hot water storage is that is 
generally has lower storage density and higher losses than thermochemical and latent systems [15].

\subsubsection{Numerical modelling of hot water storage tanks}

The performance of a hot water storage tank is governed by several time-dependent equations that account for conservation of mass, energy and momentum. When modelled in a simulation environment like TRNSYS, these equations are solved numerically rather than analytically. This is done by discretizing the governing differential equations, which are then solved iteratively within a specified convergence tolerance [4]. Discretization occurs in time (the simulation is divided into time steps) and space (the tank is divided into nodes).

One challenge with hot water tanks is the natural tendency for water to equilibrate its temperature. As the average temperature of the water rises, further addition of heat, or charging, becomes more difficult. Additionally, as stored heat disperses through the water the quality of the heat is reduced, reducing the amount of useful energy that can be provided or discharged. These challenges are partly overcome by promoting stratification of the water, whereby the equilibration of water temperature is impeded so that there exists a higher temperature region in the tank that can provide high quality heat to support a load, and a lower temperature region in the tank that can be more effectively charged [15]. Hot water storage tanks are naturally capable of some extent of stratification, as the lower density of hotter water will slow the mixing with colder and denser water at the bottom of the tank (ignoring the inversion of this trend around $0{ }^{\circ} \mathrm{C}-4{ }^{\circ} \mathrm{C}$ where the density of water slightly increases with temperature). In systems where heat exchange with the water to charge and discharge the tank occurs outside of the tank, this effect is typically enhanced 
by ensuring that lower temperature water from the bottom of the tank is drawn for charging to the energy source, and the higher temperature water returns near the top portion of the tank, and vice versa for discharging. Where charging or discharging occur through heat exchange internal to the tank, stratification can be similarly promoted through proper selection of the location and flow direction of the heat exchangers. Some systems try to further enhance tank stratification using physical mechanisms like baffles [15]. Because stratification has an important effect on the performance of a hot water storage tank, it is important to consider during modelling.

The hot water storage tank simulations conducted in this study are modelled using the TRNSYS type 534 from the storage tank library [16]. This type is used to model a stratified, fluid-filled tank with a constant volume and vertical orientation. The tank is divided into a number (selected by the user) of discrete isothermal nodes with equal volume. At each simulation timestep the tank model accounts for the following parameters:

- Flow of water entering and leaving the tank through a user-specified number of inlets and outlets.

- Heat transfer to and from the tank's surroundings, based on the surrounding temperature and a user-specified thermal conductivity of the tank material.

- Thermal conduction and physical mixing occurring between the tank nodes.

- Any other heat transfer from user-specified heat exchangers or auxiliary heaters. 


\subsection{The Fourier transform}

\subsubsection{Definition}

The Fourier transform is a mathematical process that transforms a real-valued function, $F(t)$, into a complex-valued function, $\Phi(v)$, and is defined as

$$
\Phi(v)=\int_{-\infty}^{\infty} F(t) e^{-2 \pi i v t} d t=\mathcal{F}(F(t)) .
$$

This transformation is reversible through the inverse Fourier transform

$$
F(t)=\int_{-\infty}^{\infty} \Phi(v) e^{2 \pi i v t} d v=\mathcal{F}^{-1}(\Phi(v)) .
$$

While in general $t$ and $v$ represent abstract variables, the Fourier transform is often used to transform a function from the time domain to the frequency domain, in which case these variables represent time and frequency respectively [17]. In this way the Fourier transform allows one to decompose a residual heating profile (a function in the time domain) into its constituent frequencies. Given that the transformed function in the frequency domain is complex, it can be expressed in polar form as

$$
\Phi(v)=a(v) e^{i \phi(v)}
$$

where the modulus, $a$, and the argument, $\phi$, give the amplitude and phase, respectively, of the underlying sinusoid with frequency $v$.

\subsubsection{Discrete Fourier transform}

The Fourier transform as defined above in Eq. (1) invokes integral calculus and so requires an analytical expression of the integrand, $F(t)$. Often no analytical expression is available, and the integrand is instead defined by discretized experimental data points. The need to 
apply Fourier transforms to such discretized data lead to the development of a compatible discrete Fourier transform (DFT).

A sequence of $N$ values in the time domain, $\left\{x_{0}, x_{1}, \ldots, x_{N-1}\right\}$, can be transformed to a related sequence of $N$ values in the frequency domain, $\left\{y_{0}, y_{1}, \ldots, y_{N-1}\right\}$, through a DFT defined by

$$
y_{k}=\sum_{n=0}^{N-1} x_{n} e^{\frac{-2 \pi i k n}{N}}
$$

Like the continuous case, the DFT can be reversed by an inverse transform expressed as

$$
x_{n}=\frac{1}{N} \sum_{k=0}^{N-1} y_{k} e^{\frac{2 \pi i k n}{N}} .
$$

In practice the DFT is typically computed in matrix form [17] by the expression

$$
\left[\begin{array}{c}
y_{0} \\
y_{1} \\
\vdots \\
y_{N-1}
\end{array}\right]=\left[\begin{array}{ccccc}
1 & 1 & 1 & \ldots & 1 \\
1 & e^{-2 \pi i / N} & e^{-4 \pi i / N} & \ldots & e^{-2(N-1) \pi i / N} \\
\vdots & \vdots & \vdots & \vdots & \vdots \\
1 & e^{-2(N-1) \pi i / N} & e^{-4(N-1) \pi i / N} & \ldots & e^{-2(N-1)^{2} \pi i / N}
\end{array}\right]\left[\begin{array}{c}
x_{0} \\
x_{1} \\
\vdots \\
x_{N-1}
\end{array}\right] .
$$

\subsubsection{Fast Fourier transform}

The fast Fourier transform (FFT) simply refers to a specific class of algorithms commonly used to compute a DFT in a more computationally efficient manner. Computational efficiency is often described through a notation like $O(f(n))$, which indicates that as the size of the input to undergo some computation increases in size by $n$, the time taken for the computation increases according to the relationship $f(n)$. For example, an efficiency of $O\left(n^{2}\right)$ means that as the input increases in size by $n$ the computation time increases by the square of $n$, which results in very large computation times for large inputs. 
The most prevalent FFT algorithm is the Cooley-Tukey algorithm [18], although several others exist. A simple DFT calculation directly using Eq. (4) would have a computational efficiency of $O\left(n^{2}\right)$ because a vector of $n$ values would require $n$ transformations, each of which requires the summation of $n$ terms. The FFT algorithms, as the name implies, perform the same DFT faster with an efficiency of $O(N \log N)$. This is accomplished purely through mathematical manipulation of the matrix multiplication process shown in Eq. (6) (specifically through a clever factorization of the large matrix of exponentials) which reduces the number of computations required without altering the result. Because the FFT is the standard method used to compute a DFT, the two terms are often used interchangeably.

\subsection{Mathematical optimization concepts}

\subsubsection{Optimization problems and dimensionality}

Optimization problems consist of one or more objectives that are to be minimized or maximized. The objectives are dependent variables that are related to independent variables mathematically through one or more objective functions. The goal in any optimization problem is to decide the values of the independent variables that provide the optimal objective function value; hence the independent variables are often called decision variables. Often there are other conditions that the solution must satisfy, called constraints, that are not directly related to optimizing the objective, however they place restrictions or bounds on the combinations of decision variables that are considered feasible. The following is an example of a generic constrained optimization problem:

$$
\min z=f\left(x_{1}, x_{2}\right)
$$


Subject to

$$
\begin{aligned}
& h_{1}\left(x_{1}, x_{2}\right) \geq c_{1} \\
& h_{2}\left(x_{1}, x_{2}\right)=c_{2} \\
& x_{1} \geq 0, x_{2} \geq 0
\end{aligned}
$$

Here Eq. (7) represents the objective function, where the objective is to minimize some quantity $z$ which is a function of the decision variables $x_{1}$ and $x_{2}$. Eq (8) and Eq. (9) represent constraints on the problem, where $h_{1}$ and $h_{2}$ are some functions of the decision variables and $c_{1}$ and $c_{2}$ are constants. Constraints are distinguished as either inequality constraints (like Eq. (8)) or equality constraints (like Eq. (9)) based on the type of operator in the constraint because they are generally handled differently when the problem is solved. Finally, Eq. (10) also presents constraints on the decision variables, however direct constraints on the value of individual decision variables are more often called variable bounds instead of constraints.

The optimization problem above has a single objective. Other problems may have multiple objectives and are hence called multi-objective. In such problems there usually exists a trade-off between the various objectives. These types of problems are more complex than single objective problems and are typically solved in two steps. First a set of pareto optimal solutions is determined. These are the solutions where no objective can be improved without worsening the value of another objective. Next a subjective decision is made between the pareto optimal solution to select the final solution.

The above problem has multiple decision variables, so it is considered a multivariate problem, where the alternative would be a univariate problem with a single decision variable. Much like increasing the number of objectives in an optimization 
problem, increasing the number of decision variables has the effect of complicating the solution of the problem. Multivariate problems require significantly more computational resources to solve than univariate problems: as the number of decision variables grows linearly the solution space to an optimization problems grows exponentially, a challenge that is often referred to as the "curse of dimensionality" [19]. Reducing the dimensionality of a problem, whenever possible, is a good practice as it makes any problem easier to solve, and easier to understand.

\subsubsection{Optimization methods}

There are many different methods used to solve optimization problems. The choice of method is influenced by the nature of the problem:

- When the objective function or constraints cannot be expressed analytically, then numerical techniques are required.

- Unconstrained problems can generally be solved much more easily than constrained problems.

- If any of the objective function(s) or constraints are non-linear in form, then more sophisticated solution methods are required.

When an analytical expression is available for the objective and the problem is unconstrained, then traditional differential calculus can be used to solve the problem directly. In some simpler cases, a constrained problem can be converted to an unconstrained problem as is the case when applying Lagrangian multipliers. More generally, however, a mathematical programming problem needs to be defined (like that which is shown in Eq. (7) through Eq. (10)) for constrained problems and solved using an appropriate algorithm. There are many different algorithms that have been proposed to 
solve different classes of mathematical programming problems (e.g. linear, integer, mixed integer, non-linear and dynamic). For example, the best-known algorithm for linear programming problems is the simplex method.

Often the problem of interest cannot be neatly defined using analytical expressions, for instance when the objective function is related to the output of a complex simulation. In such cases it is common to employ purely numerical methods to find the optimal solution. These methods differ from the algorithms employed in traditional programming problems in that they do not require any information regarding the actual analytical expression of the objective function, rather they simply require the ability to evaluate the objective function for any combination of decision variables. The algorithm then attempts to perturb the decision variables in a predefined manner to improve the value of the objective function until the stopping criterion is met, indicating that the optimum has been found.

A significant challenge in optimizing complex, non-linear systems is the difficulty of avoiding local optima that are in general not equal to the global optimum. In these cases, solver algorithms can be sensitive to the starting point, and so a multi-start approach is used where the algorithm is repeated for many starting points. An alternative approach is to use a heuristic optimization method that has a built-in property allowing it to search the entire solution space without being overly susceptible to local optima. One such method that is used in this study, the genetic algorithm, is briefly described in the following section.

\subsubsection{Genetic algorithms}

Genetic algorithms are a popular form a numerical optimization method commonly used for complicated problems. They are particularly useful for their ability to "escape" local 
optima, as well as their ease of implementation for problems whose dynamics may otherwise be complex. In a genetic algorithm an initial population of solutions is randomly generated. Each solution, or individual, consists of an ordered vector of the decision variables' values. The population of solutions is then subjected to several reproductive cycles or generations until a stopping criterion is met. In each generation the objective function, or fitness function, for each individual is calculated, and a certain proportion of the individuals are randomly selected to survive to the next generation (eliminations are made at random, where the individuals with lower fitness are more likely to be removed). The remaining individuals "reproduce" to replace those that were eliminated by generating new solutions vectors that are recombinations of the surviving individuals' decision variable values. This process is often called crossing-over. The values of each decision variable for each individual are then given a certain probability of mutating, or randomly changing - this is an essential step for the algorithm to avoid being trapped in local optima. Once a new population is generated, fitness functions are re-evaluated and the cycle repeats until a stopping criterion is met. For example, one commonly used stopping criterion is when there has been no improvement in the population's average fitness value over a certain number of generations. This process is summarized in Figure 2.1. 


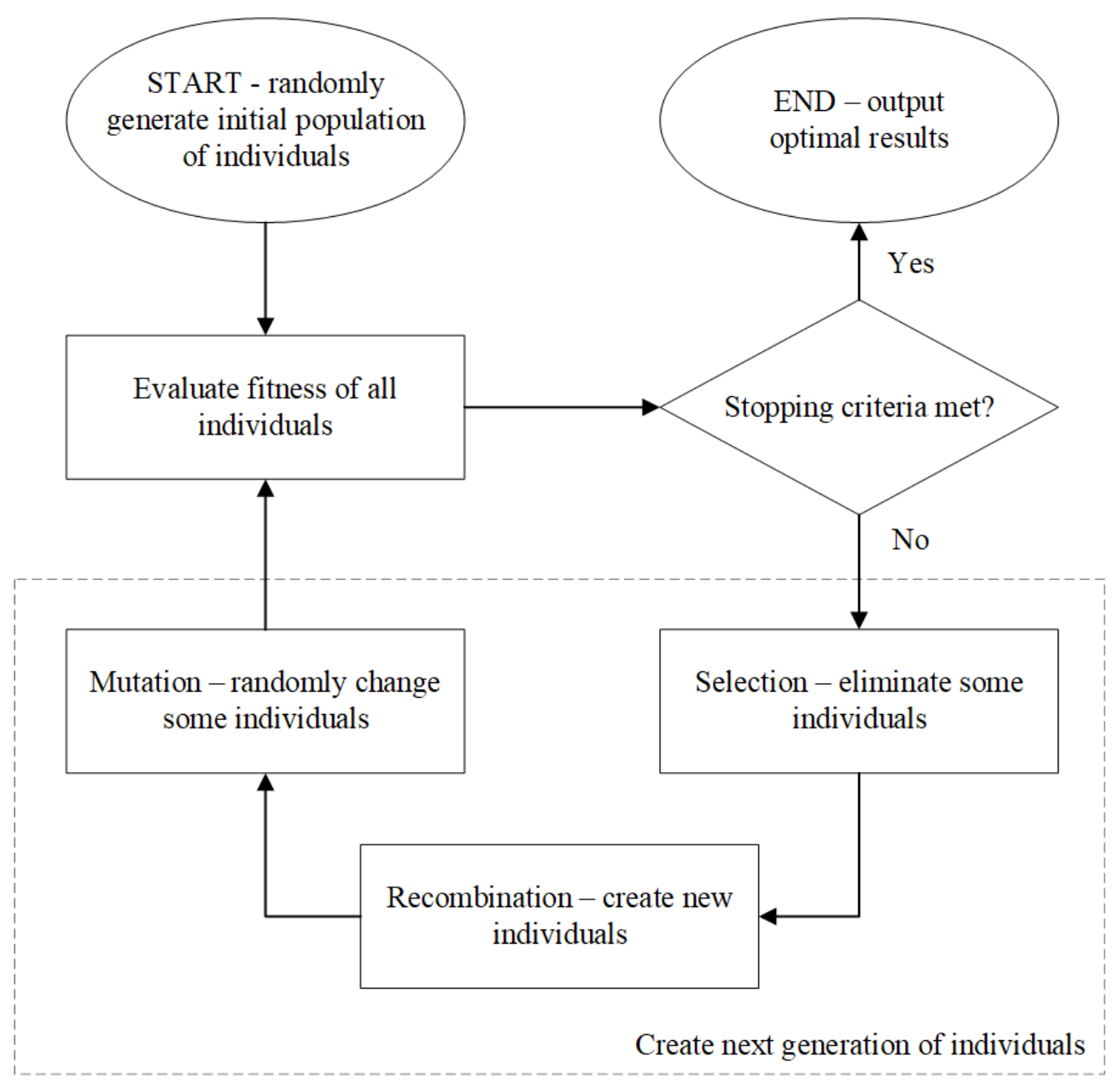

Figure 2.1: Flow diagram depicting the general steps of a typical genetic algorithm optimization method.

\subsection{Regression analysis}

The sizing method developed in this thesis relies on ordinary least-squares (OLS) regression techniques. Regression analysis involves determining the best mathematical model to relate some response (dependent) variable to a set of predictor (independent) variables. The variables are so named because the model is intended to predict the response of the system provided with some independent inputs. Regression can be split into two separate parts: determining the best type or form of the model and determining the values of any parameters (constants) in the model. 
The term "least-squares" refers to the approach taken to determine the model parameters: the parameters are selected to minimize the sum of the squared model residuals. This is not the only method available for parameter estimation, but it is by far the most common. Other methods include minimizing the absolute residuals (not commonly used because of the trouble that absolute value functions give in differentiation) and maximum likelihood estimation (MLE) methods. MLE methods select the parameters that maximize the likelihood of the sample that is observed, and while they are generally considered more versatile to the least-squared method, they yield an identical result in many common regression problems [13]. The "ordinary" part of the regression means that it is a linear regression where the model is linear in all its parameters (although it can be non-linear in its predictor variables). The type of model used is usually selected based on how much is known about the system. Ideally a model can be formulated that represents the underlying processes occurring, although when systems are complicated or not well understood it is still possible to use exploratory techniques to find a model that will provide some predictive power of the system.

\subsubsection{Significance tests}

Once a model form is selected, least-squares or any other suitable technique is used to determine the best parameter values with the available data; however, there is no guarantee that this best model is a good one. Generally additional tests for statistical significance are used to decide if the selected model is appropriate for the situation. One common test to check the significance of the overall model is the F-test or F-statistic, which is a measure of the ratio of variance explained by the regression model to the variance not explained by the model [13]. The F-test consists of choosing a critical F-statistic value based on the users 
desired level of confidence in the model - commonly chosen as $95 \%$ - and accepting the model if the calculated F-statistic exceeds the critical value. The confidence level is normally described through a corresponding $\mathrm{p}$-value, where the $\mathrm{p}$-value is a measure of the probability of error in rejecting the null hypothesis (in this case the null hypothesis is that the model is not significant). A p-value of 0.05 corresponds to a $95 \%$ confidence level, and a p-value less than $0.05(\mathrm{p}<0.05)$ would indicate the model is significant.

It is possible that even when the overall model is significant, one or more of the estimated parameters may not be. Separate tests are used to check the statistical significance of the parameters, with the most common being the t-statistic or t-test [13]. The t-test is similar to the F-test, in that a critical t-statistic value is selected based on the desired confidence level. Again, generally $95 \%$ confidence or $p<0.05$ is used, though in this case the null hypothesis is that the value of the parameter is zero. A common regression technique in cases where the number of significant parameters is not known beforehand is called stepwise regression. In stepwise regression, model parameters are progressively added or removed from the model until there are as many parameters included in the model as possible, while maintaining that each parameter is significant.

\subsubsection{Other modelling options}

OLS is used in this thesis as the model building approach for both simplicity and transparency for the user. It should be acknowledged that more sophisticated methods like machine learning could similarly be employed to potentially provide greater predictive power, at the cost of familiarity for the general engineering practitioner. The raw data used for the OLS analysis in this thesis will be made available as a supplemental data file for this purpose. 


\section{Residual heating profile analysis}

Several conventional heating systems operate on the principle of integrating time-varying heating sources and loads. Two examples of such systems include a solar thermal system for DHW heating, and a CHP heat recovery system for space heating (SH). Figure 3.1 depicts normalized source and load profiles for each of these systems over a 24-hour period on May $10^{\text {th }}$. The profiles are arbitrarily selected from datasets taken over several years and are normalized by dividing each profile by its highest value, so that the values range from 0 to 1 . For both systems, a distinct temporal mismatch can be observed between profiles, which is indicative of inefficient system operation as either heat is available at times when it is not required, or alternatively, heat is not available at times when it is required. In either case, system performance can be increased by the addition of hot water storage capacity. To better characterize the temporal mismatch between heating source and load profiles in any given system, and thereby determine the hot water storage capacity that is required, the residual heating profile needs to be determined. 


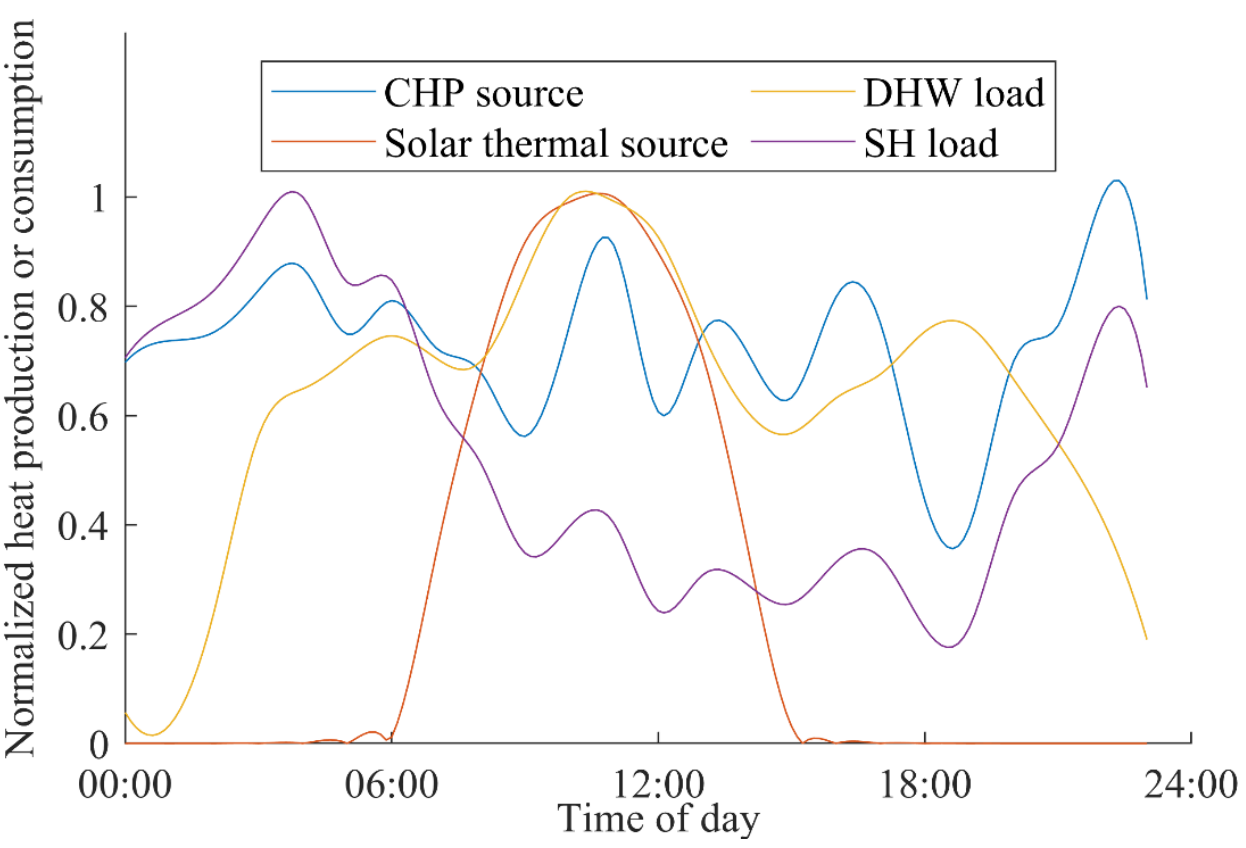

Figure 3.1: Normalized source and load profiles corresponding to solar thermal [20], and CHP [21]; and DHW [22], and SH [23] systems, respectively, over a 24-hour period on May $10^{\text {th }}$. The profiles are arbitrarily selected from datasets taken over several years.

\subsection{Relationship between residual heating profile and hot water storage volume}

The residual heating profile is obtained by subtracting the heating load profile from the heating source profile over a defined time interval. Thus, the residual is positive at times when the heating source exceeds the heating load, and negative at times when the heating load exceeds the heating source. The actual form that the residual heating profile takes can vary widely based on the nature of the heating source and load. To illustrate, Figure 3.2 depicts two different hypothetical residual heating profiles: a flat profile and a sinusoidal profile. The former is constant in time with a value of zero indicating that the heating source and load profiles are identical. No hot water storage tank is required in this case. The latter, on the other hand, varies sinusoidally with amplitude $a$, period $p$, and phase, $\phi$. A hot water 
storage tank would be required in this case to store heat during periods when the residual is positive, and discharge heat during periods when the residual is negative.

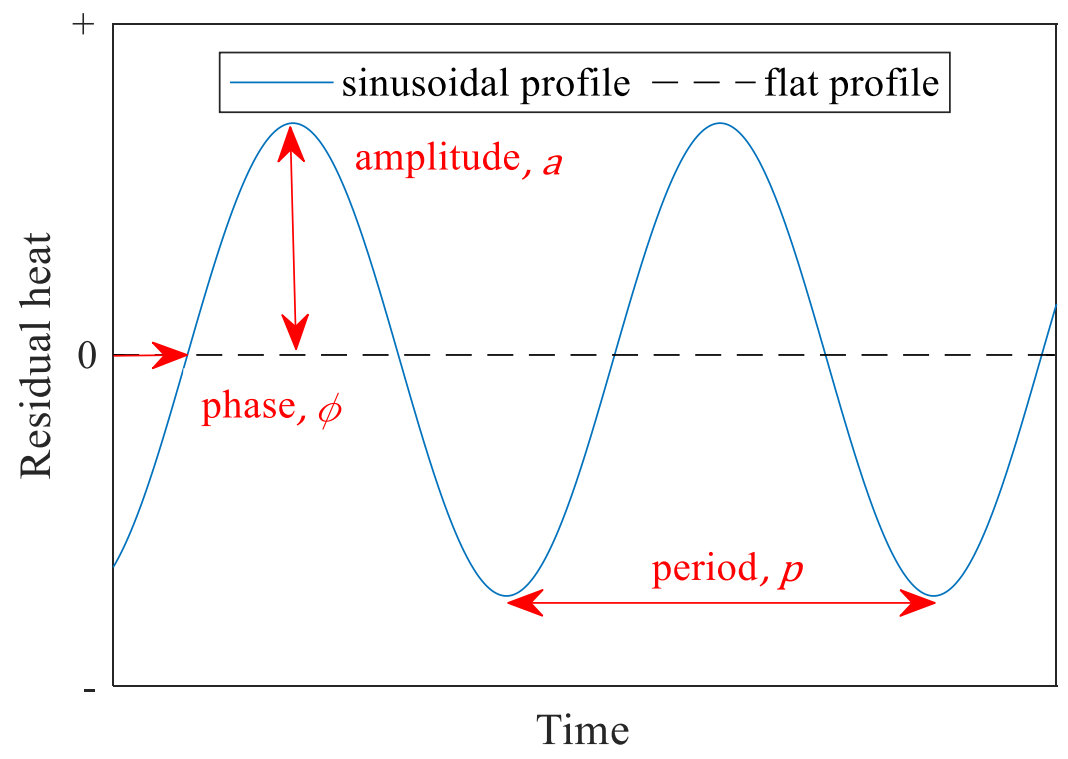

Figure 3.2: Two hypothetical residual heating profiles. Sinusoidal profiles can be characterized using their characteristic amplitude, period and phase.

The residual heating profile thus dictates when the storage tank can be charged and discharged, and it is this fundamental relationship between residual and storage that suggests that a RSM can be developed to link the two together. As the residual heating profile of any given system can be highly complex, there is a need to simplify it as much as possible. This simplification can be achieved by carrying out a FFT waveform conversion. FFT algorithms provide a convenient way to calculate the Fourier transform of a residual profile, decomposing it into a set of simple sinusoidal functions each characterized by an amplitude, a period (or frequency) and a phase. In this way the Fourier transform allows one to separate the periodic parts of a residual profile from the chaotic noise. It is assumed that the periodic parts of the residual would interact with a hot water storage tank in a predictable way (e.g. repeated charge and discharge cycles), which in turn 
is what suggests that they may be suitable predictor variables for the optimal storage tank volume.

The result of a FFT is qualitatively demonstrated in Figure 3.3 which shows a hypothetical residual profile (Figure 3.3a) that is decomposed and repackaged into amplitude (Figure 3.3b), and phase (Figure 3.3c) profiles, both of which are in the frequency domain. A FFT algorithm developed by Cooley and Tukey [18] is used in the current study to decompose arbitrarily complex residual heating profiles, $r(t)$, into their characteristic amplitudes, periods, and phases. These residual characteristics are subsequently used as predictor variables for developing RSM functions of the form

$$
\widehat{V}_{\text {opt }}=f(\vec{a}, \vec{p}, \vec{\phi})=f(r(t))
$$

which are used to determine an estimate, $\widehat{V}_{\text {opt }}$, of the optimal hot water storage tank volume, $V_{\text {opt }}$.

(a)

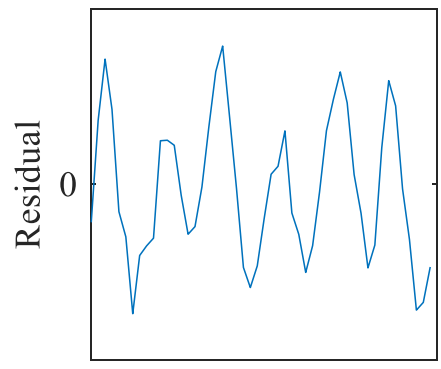

Time (b)

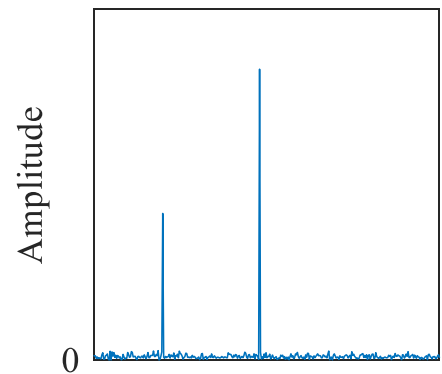

Frequency (c)

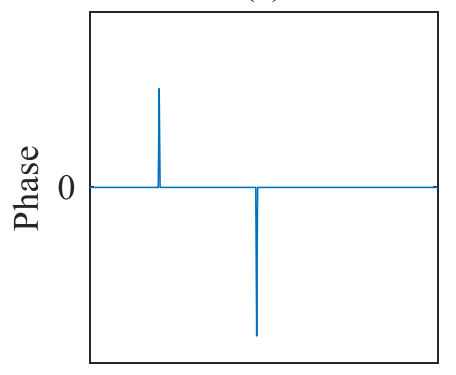

Frequency

Figure 3.3: A hypothetical residual heating profile composed of two sinusoids (and noise) is shown in (a). Using the FFT algorithm, the profile is decomposed into the two underlying sinusoids; the amplitudes and phases are plotted against frequency in (b) and (c), respectively. 


\subsection{Residual heating profile generation}

A randomized set of 150 residual heating profiles, $r(t)$, of varying complexity is generated using the Matlab ${ }^{\circledR}$ software for each system configuration considered in this study. This number was selected arbitrarily to provide enough samples for meaningful regression, and to limit computational intensity at a reasonable level. Each profile is in units of $\mathrm{kW}$, and is constructed from $n=5$ sinusoids using the following expression:

$$
r(t)=\text { noise }+\sum_{i=1}^{n} a_{i} \sin \left(2 \pi f_{i} t+\phi_{i}\right)
$$

where $t$ represents time, and $a_{i}, f_{i}$, and $\phi_{i}$ represent the amplitude, frequency, and phase of sinusoid $i$, respectively. Both the rationale used for limiting $n$ to five, and the methods used to generate noise for each profile are described in Chapters 7 and 8.1, respectively. Further to substituting the frequency term in Eq. (12) with a period term as follows:

$$
f_{i}=\frac{1}{p_{i}},
$$

a random number generator is used to select values for $a, p$, and $\phi$ that are drawn from uniform distributions. In the current study, these values are limited to the following predetermined ranges: $0-250 \mathrm{~kW}$ for amplitude (real number values); $0-2 \pi$ for phase (real number values); and $8-48$ hours for period (integer values). An upper limit of $250 \mathrm{~kW}$ is chosen for amplitude as many small and medium sized thermal systems fall below this value. A lower limit of 8 hours is chosen for period as most thermal profiles examined exhibit little variation below this value. An upper limit for period of 48 hours is chosen because the focus of the study is on short term storage rather than seasonal storage. The residuals are generated for a year and are assumed to have a mean of zero, which implies that over this time period, the supply energy is equal to the load energy. This assumption 
is justified as the main role of hot water storage is to match temporally shifted heating sources and loads over a specified time horizon.

In real systems, the residual may not necessarily be completely random; it may be somewhat predictable. For example, a solar thermal source or a DHW load may have very predictable diurnal profiles that vary seasonally. In general, however, this is not the case, and residual profiles can be complicated depending on the combination of source and load profiles. The residuals considered in this study are randomly generated to ensure that the method is flexible enough to deal with a wider range of residual profiles that may be encountered in practice, including those that are less conventional or predictable. 


\section{Transient hot water storage tank model}

\subsection{TRNSYS software}

A numerical model is developed using the TRNSYS tool [24] to assess the performance of a hot water storage tank of any given volume as a function of residual heating profile. TRNSYS is a commercially available transient simulation environment that comprises a vast library of components that can be linked together to form a desired system. It uses an iterative solver to determine system convergence and output results based on the users' preferences. The tool and its component models, called types, have been validated extensively in the literature [25].

Each TRNSYS type models a single component of a system. For example, TRNSYS types exist for hot water storage tanks, solar collectors, controllers, pipes, and valves. Any type is characterized by a list of parameters, inputs and outputs. Parameters are the fixed properties of a component provided by the user. A type for a hot water storage tank would include a parameter for the tank's height, which remains fixed throughout the simulation. Inputs are the list of variable values that need to be provided to a type at each time step (e.g. the tank's surrounding temperature), and the outputs are the values that are calculated by the type throughout the simulation (e.g. the heat loss to the environment). Built into each type are the set of equations that govern the behaviour of that component (e.g. heat transfer processes), which use the tank's fixed parameters and variable inputs to calculate the outputs at each time step. A TRNSYS model is a collection of types that are connected to exchange information between each other; the outputs of one type are passed to another type and used as inputs. Connections are chosen in a way that makes the simulated system 
mimic the behaviour of the real system being modelled. The governing equations for each type are iteratively solved (with numerical methods) during a timestep until the system of equations converges on a solution. The model repeats this converging process for each time step until the end of the simulation is reached. An external input or boundary condition provides the forcing required to drive the simulation.

In this thesis, the system being modelled is a simplified thermal system with an arbitrary heat source and load (i.e. they are modelled by general types that represent heat sources and sinks, rather than as types for specific real components like solar collectors or heat exchangers), that are buffered by a hot water storage tank. The simulation is driven by an imposed residual profile, which dictates the behaviour of the hot water storage tank.

\subsection{Model description}

The model comprises a hot water storage tank, a simplified source and load, a cooling tower, and an auxiliary heat source as shown in Figure 4.1. The system is closed with water as the only working fluid, which flows through pipes that are depicted as arrows. Fluid is driven by variable speed pumps (VSP), and flow streams interact at various mixing valves (MV) and modulating control valves (CV) to maintain specified temperatures and flow directions. The cooling tower dissipates excess heat at times when the source exceeds the load, and the storage tank is full; and the auxiliary heater supplies back up heat at times when the load exceeds the source. The hot water storage tank can only be charged or discharged at any given time step. Time series simulations are conducted using a 1-hour time step with a 10-node discretization of the storage tank. Although both sub-hourly time steps, and increased discretization of the storage tank were initially investigated, these measures were found to be undesirable as computational intensity increased considerably 
for a negligible associated decrease in error. Moreover, a 1-hour time step was found to be preferable as data at this temporal resolution is most common among practitioners [5].

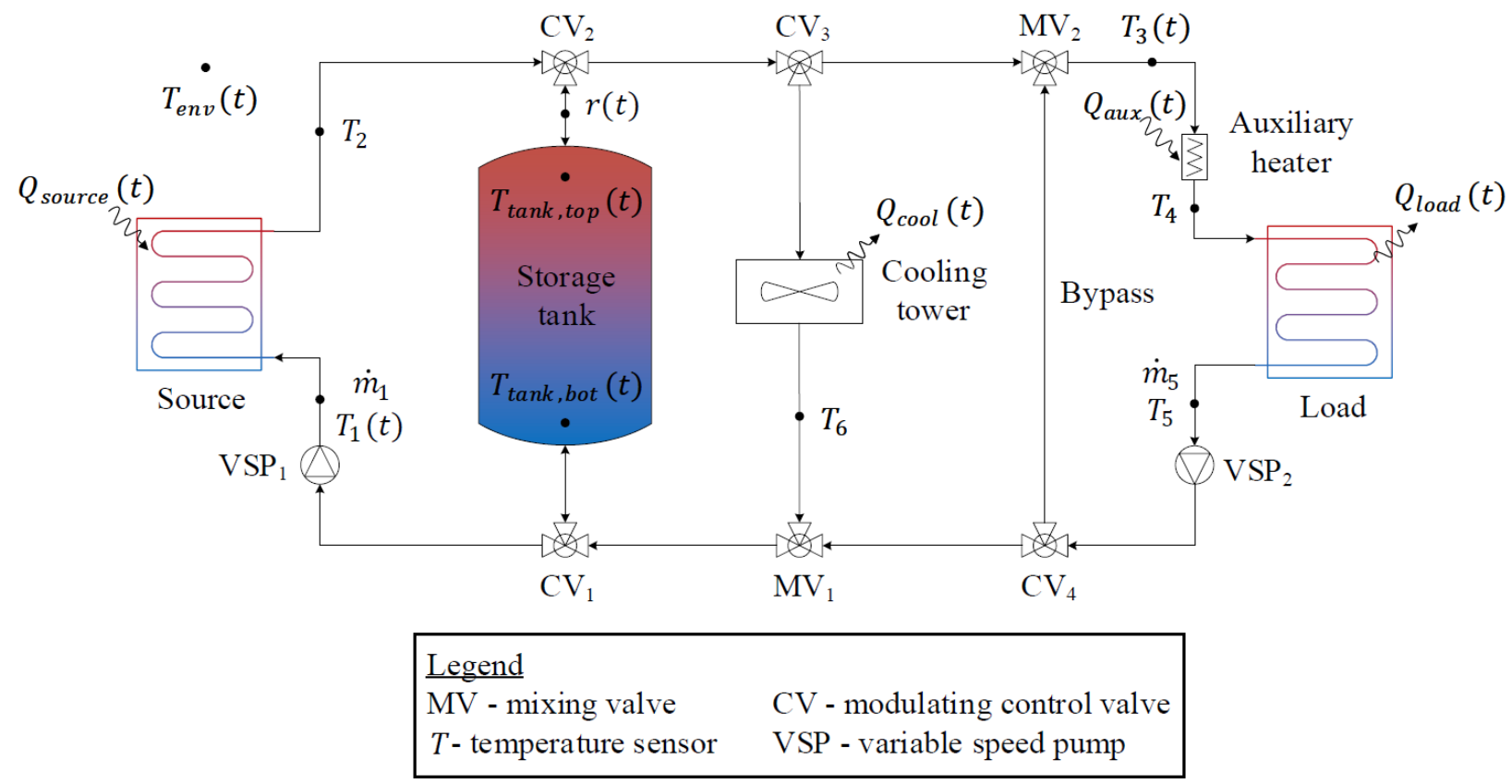

Figure 4.1: Schematic of transient numerical model developed [24] to assess the performance of a hot water storage tank of any given volume as a function of residual heating profile.

Variable model inputs include the residual heating profile, $r(t)$, and the outdoor environmental temperature, $T_{e n v}(t)$. Fixed model inputs include the hot water storage tank volume, and system temperatures $T_{2}, T_{4}, T_{5}$, and $T_{6}$. Data for all other variables depicted in Figure 4.1 are output by the model. If at any given time step $r(t) \geq 0$, and the tank is not full, then the tank is charged by the source. To provide the desired heat input into the tank, the mass flow rate of $\mathrm{VSP}_{1}, \dot{m}_{1}$, is adjusted according to

$$
\dot{m}_{1}=\frac{r(t)}{C_{p}\left(T_{2}-T_{1}(t)\right)}
$$

where $C_{p}$ represents the specific heat capacity of water. The tank is deemed full when either the uppermost tank temperature, $T_{\text {tank,top }}$, exceeds $95^{\circ} \mathrm{C}$, or when the bottommost tank 
temperature, $T_{\text {tank,bot }}$, is within a $10{ }^{\circ} \mathrm{C}$ range of $T_{2}$. In such instances, any excess heat is dissipated via the cooling tower. If on the other hand $r(t)<0$ at any given time step, and $T_{\text {tank,top }}(t)>T_{5}$, then the tank is discharged to service the load. To provide the desired heat output to the load, the mass flow rate of $\mathrm{VSP}_{2}, \dot{m}_{5}$, is adjusted according to

$$
\dot{m}_{5}=\frac{-r(t)}{C_{p}\left(T_{4}-T_{5}\right)}
$$

where the negative signed is used to make the calculated mass flow rate positive. The flow rate calculated in Eq. (15) is then split at $\mathrm{CV}_{4}$ based on the state of the tank, with the goal of making $T_{3}(t)=T_{4}$. If $T_{3}(t)>T_{4}$, the flow through the bypass line is increased to temper the hot fluid to the desired service temperature, $T_{4}$. When the temperature of the tank is low then it may be that no distribution of flow at $\mathrm{CV}_{4}$ will achieve $T_{3}(t)=T_{4}$. In this case the flow is split at $\mathrm{CV}_{4}$ to maximize $T_{3}(t)$ (to bring it as close to $T_{4}$ as possible), and if it remains that $T_{3}(t)<T_{4}$, then the auxiliary heater provides the additional heat required to bring the fluid to the desired service temperature $\left(T_{4}\right)$. The following assumptions are used in formulating the model:

1. Liquid water is the working fluid and storage medium;

2. Constant thermophysical properties of water at $50^{\circ} \mathrm{C}$ are assumed over the temperature range considered;

3. The hot water storage tank is a stratified vertical tank of cylindrical shape (TRNSYS type 534) with an edge loss coefficient that varies as a function of the selected tank insulation thickness. Details regarding the hot water storage tank geometry and thermophysical properties are discussed in Chapter 5; 
4. The hot water storage tank operates at atmospheric pressure, and at temperatures between $5{ }^{\circ} \mathrm{C}-95{ }^{\circ} \mathrm{C}$. If $T_{\text {tank,bot }}$ decreases below this lower threshold temperature, an auxiliary heater inside the tank turns on to bring this value within operating range;

5. The cooling tower operates with a fixed temperature drop of $T_{2}-T_{6}=15^{\circ} \mathrm{C}[26]$;

6. The auxiliary heater and cooling tower operate at $100 \%$ efficiency;

7. The temperature drop across the load is fixed at $T_{4}-T_{5}=11^{\circ} \mathrm{C}$ [27]. In order to impose a residual on the system, either a constant flow is required with a variable temperature drop, or a constant temperature drop is required with a variable flow (if both parameters are variable the system becomes more difficult to model). Because the nature of the residual in this study is random, it is difficult to select a fixed flow rate in advance of a simulation that would provide realistic temperatures at the load, which is why a fixed temperature drop is assumed;

8. Pumping energy requirements, and pressure losses in valves are not included;

9. Heat losses in pipes are not included.

Prior to using the simulation model in the analysis, a manual verification of mass and energy balances under varying system conditions was carried out to ensure the model operates as intended. 


\section{Optimization framework}

An optimization framework is developed to determine the optimal hot water storage tank volume for any given residual heating profile. The framework is based off of a method developed by Jones [28] and involves running an optimization algorithm in the Matlab ${ }^{\circledR}$ environment which interacts with the transient hot water storage tank model developed in TRNSYS. As shown in Figure 5.1, for each input residual heating profile, multiple tank volumes are evaluated via simulation, and tank performance is quantified using a costbased objective function. Simulations are run in an iterative manner following the optimization algorithm until the solver determines that the objective function is minimized.

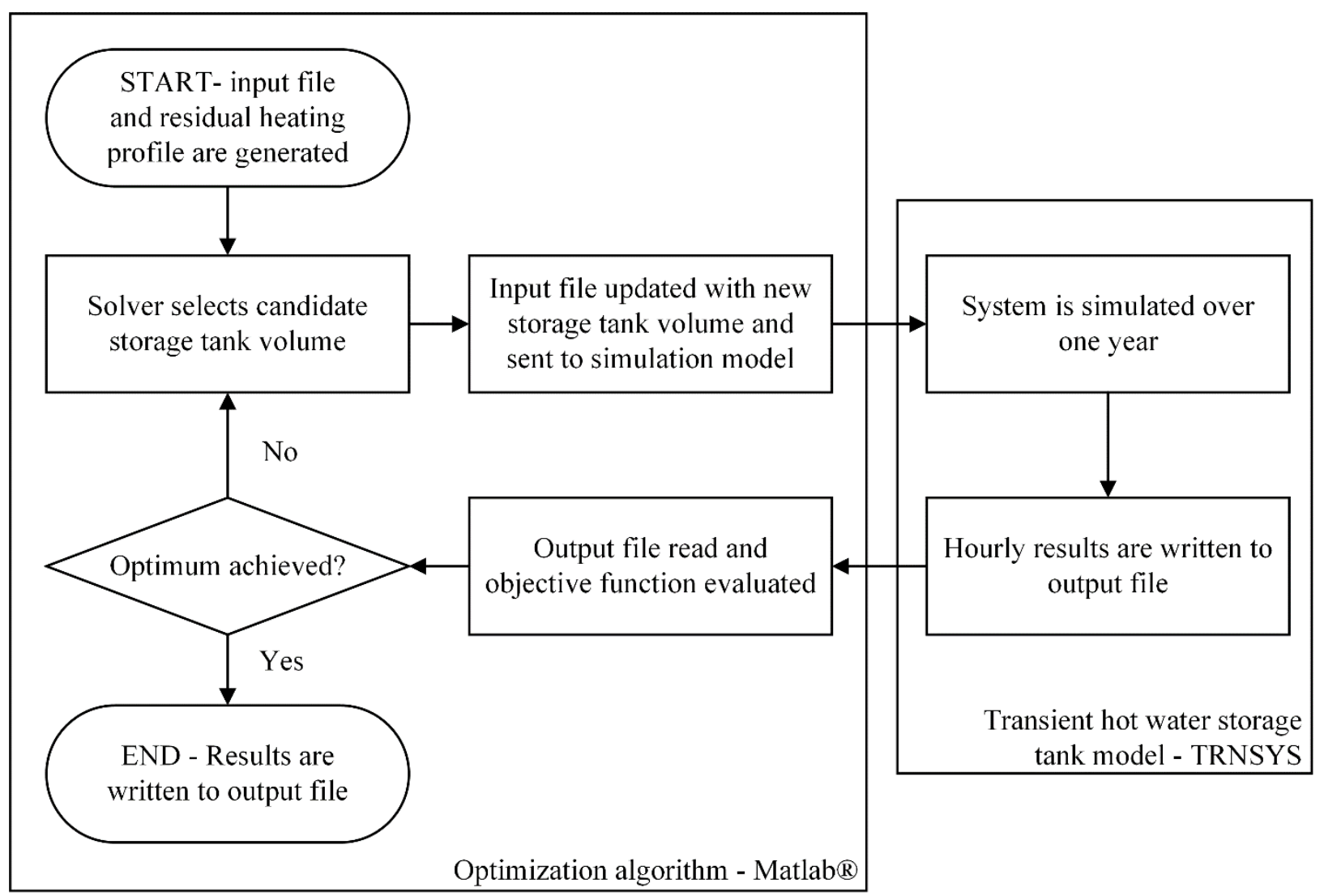

Figure 5.1: Optimization framework used to determine optimal hot water storage tank volume for any given residual heating profile. 


\subsection{Objective function}

The objective function for any given residual heating profile is defined as the hot water storage tank volume, $V_{\text {opt }}$, that minimizes the system net present cost, $N P C$. The $N P C$ is given by

$$
N P C=C_{c a p}+C_{\text {ins }}+C_{\text {cool }}+C_{\text {aux }}+C_{\text {maint }}+C_{\text {energy }}
$$

where the variables on the right-hand side, in the order stated, represent the capital cost of the storage tank, the cost of tank insulation, the cost of the cooling tower (used to reject excess heat to the environment), the cost of the auxiliary water heater, the tank maintenance cost, and the cost of the energy produced by the auxiliary heating unit. Most of these variables represent fixed costs except for the latter two which are functions of the project lifetime, $Y$, and can be expressed as

$$
C_{\text {maint }}=\sum_{y=1}^{Y} \frac{M F \times C_{c a p}}{(1+i)^{y}}
$$

and

$$
C_{\text {energy }}=\sum_{y=1}^{Y} \frac{C_{\text {energy }, a}}{(1+i)^{y}},
$$

respectively, where $M F$ is the maintenance factor that relates maintenance costs to capital cost, $i$ is the discount rate, and $C_{\text {energy, } a}$ is the annual energy cost. $C_{\text {energy, }}$ is calculated as

$$
C_{\text {energy }, a}=E_{\text {annual }} \times r_{E}
$$

where $E_{\text {annual }}$ represents the annual auxiliary energy that is supplied to the system (calculated via simulation), and $r_{E}$ is the cost of auxiliary energy per unit consumed. 
System components include a steel cylindrical water tank insulated with mineral fiber insulation; an electrically driven auxiliary water heater; and an axial-fan, induceddraft cooling tower. The auxiliary heater and cooling tower are sized to meet the maximum required hourly auxiliary heating rates and cooling rates over the course of the year, respectively. Fixed costs for all components (i.e., $C_{c a p}, C_{i n s}, C_{c o o l}$, and $C_{a u x}$ ) are taken from the RSMeans mechanical cost data 2019 handbook [29], and are shown in Table 5.1. Costs associated with peripheral components such as circulation pumps, heat exchangers, and piping were neglected in the objective function, as these components are assumed to be identical between all cases. Apart from fixed costs, an annual maintenance factor, discount rate, and project lifetime of $3 \%, 5 \%$, and 10 years, respectively are assumed in the current study [30], [31].

The objective function presented in Eq. (16) captures the underperformance due to under- and over-sizing of the hot water store tank as follows:

- If the tank is under-sized (smaller than optimum) then the hot water storage tank won't be able to store enough heat during the periods of higher source input, which will lead to higher consumption of backup auxiliary energy during peak load periods. This will also require larger and more expensive cooling and auxiliary heating components.

- If the tank is over-sized (larger than optimum), then a larger up front cost is paid for the tank. Additionally, the quality of stored heat tends to be degraded by the larger volume of water, so that the tank may not be able to provide useful heating when the load increases. 
- Somewhere in between these extremes lies the optimal storage volume, where the NPC of the system is at a minimum.

Table 5.1: Component fixed unit costs used to quantify the objective function.

System component $\quad$ Fixed unit cost [29]

Steel water storage tank (height to $330 \mathrm{USD} / \mathrm{m}^{3}$ storage capacity diameter ratio greater than 1)

Axial fan, induced draft cooling tower 142 USD/ton cooling capacity (40.6 $\mathrm{USD} / \mathrm{kW}$ cooling capacity)

Auxiliary electric hot water heater $36 \mathrm{USD} / \mathrm{kW}$ heating capacity

Mineral fibre insulation, thermal $\left\{\mathrm{USD} / \mathrm{m}^{2}\right\}=3.6 \mathrm{t}+34.2$, conductivity of $0.038 \mathrm{~W} / \mathrm{mK}$ at $50{ }^{\circ} \mathrm{C} \quad$ where $\mathrm{t}=$ thickness in $\mathrm{cm}$

\subsection{Decision variables}

A series of preliminary multivariate optimizations were conducted at the onset of this study to assess the sensitivity of the objective function to several storage tank variables other than the volume. These variables include the storage tank height to diameter ratio, insulation thickness, and inlet height on the discharge loop. The latter was considered as the temperature distribution in the tank is dependent on both the entry height and temperature of the returning fluid to the tank. As little sensitivity to these variables was shown from the preliminary optimizations, the variables were removed from the objective function and treated as constants, making it possible to reduce the complexity of the problem from a multivariate to a univariate optimization problem. Multivariate problems 
require more complex optimization techniques whose computational requirements were deemed prohibitive given the large number of optimizations to be conducted.

Table 5.2 shows the fixed values that are assumed for each storage tank variable considered in the current study. A tank height to diameter ratio of 1 is selected as this ratio corresponds to the smallest surface area of a cylinder for a given volume, and thus minimizes heat losses to the environment. While a larger height to diameter ratio would enhance stratification of the hot water storage tank, initial simulations showed that reducing heat losses dominated this effect. An insulation thickness of $10.2 \mathrm{~cm}$ is selected as this is the thickest insulation for which cost data is available, and preliminary simulations favoured a thicker insulation layer, again to minimize losses. The inlet position on the discharge loop is selected to enter the bottom of the tank, as this configuration ensures the highest level of stratification in the tank. Moreover, this configuration is commonly encountered in practice [32].

Table 5.2: Fixed values corresponding to each storage tank variable considered in current study.

\begin{tabular}{ll}
\hline Storage tank variable & Fixed value \\
\hline Height to diameter ratio & 1 \\
Insulation thickness & $10.2 \mathrm{~cm}$ \\
Inlet height on discharge loop & Bottom of tank \\
\hline
\end{tabular}

\subsection{Optimization algorithm}

The preliminary multivariate optimizations used to assess the sensitivity of the objective function are performed using a genetic algorithm. As the storage tank volume is deemed to 
be the only sensitive decision variable from those initially considered, a faster univariate heuristic algorithm is employed. The following method is utilized to achieve solution convergence for all optimization scenarios:

1. the objective function is first evaluated at a coarse resolution of $5 \mathrm{~m}^{3}$;

2. The interval, spanning $50 \mathrm{~m}^{3}$, with the lowest average objective function value is selected for refinement and then evaluated at a finer resolution of $1 \mathrm{~m}^{3}$;

3. The tank volume with the lowest objective function value is selected as the optimum.

While this method is generally slower than a more traditional univariate optimization approach such as a golden section search, it is generally found to be more robust. For example, using an hourly timestep results in objective functions that exhibit moderately jagged behaviour, which in turn causes traditional univariate algorithms to get stuck in local minima. 


\section{Optimization scenarios}

A total of 36 optimization scenarios are developed in the current study based on different combinations of values of the following four extraneous variables: the auxiliary energy price, the tank environment, the source temperature, and the load temperature. Table 6.1 shows the different values considered for each of these variables, and corresponding selection rationale.

These variables are selected as they are believed to be the most significant with respect to their influence on the optimal hot water storage tank volume. Moreover, adding further variables would have increased computational intensity to an impractical level. Scenario simulations are performed on a personal computer with an intel Core i5 $2.50 \mathrm{GHz}$ CPU, and 8 GB of RAM. Under these conditions, an optimization of a single residual heating profile takes approximately 6 minutes. Since each of the 36 scenarios are optimized over a range of 150 residual heating profiles, the total computation time is estimated to be roughly 22.5 days. 
Table 6.1: Values of extraneous variables used to develop optimization scenarios, and corresponding selection rationale

\begin{tabular}{|c|c|c|}
\hline $\begin{array}{l}\text { Extraneous } \\
\text { variable }\end{array}$ & Values & Rationale \\
\hline $\begin{array}{l}\text { Auxiliary energy } \\
\text { price }\end{array}$ & $\begin{array}{l}0.07,0.105 \text {, and } 0.13 \\
\text { in USD/kWh }\end{array}$ & $\begin{array}{l}\text { Prices represent the average retail } \\
\text { energy costs for the industrial, } \\
\text { commercial, and residential sectors, } \\
\text { respectively, in the USA [33]. }\end{array}$ \\
\hline $\begin{array}{l}\text { Tank } \\
\text { environment }\end{array}$ & $\begin{array}{l}\text { Indoor tank (constant } 20 \\
{ }^{\circ} \mathrm{C} \text { environment), } \\
\text { outdoor tank in a } \\
\text { relatively warm climate } \\
\text { (Los Angeles, } \\
\text { California), and outdoor } \\
\text { tank in a relatively cold } \\
\text { climate (Ottawa, } \\
\text { Ontario) }\end{array}$ & $\begin{array}{l}\text { Tank environments are selected to } \\
\text { provide a wide range of applicability to } \\
\text { the model. The indoor tank is exposed } \\
\text { to a constant } 20^{\circ} \mathrm{C} \text { environmental } \\
\text { temperature, and the outdoor tanks are } \\
\text { exposed to their respective variable } \\
\text { outdoor temperature profiles as defined } \\
\text { by typical meteorological year files } \\
\text { included in the TRNSYS weather data } \\
\text { set [20]. }\end{array}$ \\
\hline $\begin{array}{l}\text { Source } \\
\text { temperature }\end{array}$ & $75^{\circ} \mathrm{C}$ and $95^{\circ} \mathrm{C}$ & $\begin{array}{l}95^{\circ} \mathrm{C} \text { typically corresponds to sources } \\
\text { like industrial waste heat and fossil fuel } \\
\text { based CHP plants, whereas } 75^{\circ} \mathrm{C} \\
\text { typically corresponds to commercial } \\
\text { waste heat recovery, district heating, } \\
\text { and solar-thermal systems. }\end{array}$ \\
\hline $\begin{array}{l}\text { Load } \\
\text { temperature }\end{array}$ & $40^{\circ} \mathrm{C}$ and $60^{\circ} \mathrm{C}$ & $\begin{array}{l}\text { These values correspond to common } \\
\text { service temperatures for hydronic } \\
\text { space-heating and domestic hot water } \\
\text { loads, respectively. }\end{array}$ \\
\hline
\end{tabular}




\section{Development of a generalized regression model}

The regression model is developed using a simplified approach that is based on the properties of a residual heating profile comprising a single sinusoid. As the positive halfperiods of this sinusoid correspond to time periods during which the hot water storage tank is charged, and the negative half-periods correspond to time periods during which it is discharged, it follows that integrating either half-period with respect to time would yield the amount of heat, $Q$, charged and discharged during each period, respectively [6]. For a sinusoid with period $p$, frequency $f$, and amplitude $a$, the quantity of heat charged/discharged over any given half-period can be expressed as

$$
Q=\int_{0}^{\frac{p}{2}} \mathrm{a} \cdot \sin (2 \pi f t) d t=\left.\frac{-a}{2 \pi f} \cos (2 \pi f t)\right|_{0} ^{\frac{p}{2}}=\left(\frac{a}{2 \pi f}\right)-\left(-\frac{a}{2 \pi f}\right)=\frac{a p}{\pi} .
$$

If we assume that the heat transferred is adjusted using a fixed efficiency term, $\eta$, and that the heat storage capacity of the tank is linearly related to the storage volume by a fixed energy density term, $\rho_{e}$, then it is conceivable that a suitable model for $\widehat{V}_{\text {opt }}$ would be

$$
\widehat{V}_{\text {opt }}=\frac{\eta a p}{\rho_{e} \pi}
$$

Grouping constants and substituting with a single constant, $c$ (with units of $\mathrm{m}^{3} / \mathrm{kWh}$ ), Eq. (21) simplifies to

$$
\widehat{V}_{o p t}=\operatorname{cap}
$$

Eq. (22) is further generalized into a regression model that is applicable to any given residual with $n$ sinusoids as follows: 


$$
\widehat{V}_{\text {opt }}=\sum_{i=1}^{n} c_{i} a_{i} p_{i}
$$

Eq. (23) maintains the notion of sinusoid dominance where $i=1$ corresponds to the most dominant sinusoid. In the current study, Eq. (23) is truncated at $n=5$. This value was deemed appropriate after applying Eq. (23) to a number of potential source profiles from solar (TRNSYS weather file library [20]), wind [34], and waste heat recovery processes [35]; and load profiles from space heating and domestic hot water consumption [22], and observing negligible differences in results beyond $n=5$. This theory is also supported by Pinnau and Breitkopf [6] who limited their study to four dominant frequencies. 


\section{Regression analysis of optimization scenarios}

Using the described optimization framework, 150 randomly generated residual heating profiles, $r(t)$, are optimized across 36 scenarios and matched with associated optimal storage volumes, $V_{\text {opt }}$. These $150\left[r(t), V_{\text {opt }}\right]$ pairs are used to fit a RSM for each scenario using ordinary least squares (OLS) regression. The following stepwise regression approach is employed to iteratively fit each model:

1. Use OLS regression to estimate parameters $c_{1}-c_{5}$ in Eq. (23);

2. Calculate the statistical significance of the model as a whole and of each parameter using the F-statistic and t-statistic, respectively, with a critical p-value of 0.05 [13];

3. If any of the values in Step 2 are found to be insignificant, remove one of the parameters from the model by setting it to zero and return to Step 1 .

To verify each RSM and to prevent overfitting, a validation is performed by splitting the data into training sets and testing sets. Each training set comprises half of the original 150 $\left[r(t), V_{\text {opt }}\right]$ pairs for a given scenario and is used to fit its corresponding RSM. The model is subsequently used to predict the optimal storage tank volume for the remaining 75 pairs that make up the testing set.

Two separate dimensionless metrics are used to assess the performance of the regression modeling approach: the coefficient of determination, and the relative model error. The coefficient of determination, $R^{2}$, is a statistical measure of how well the regression model predictions approximate the simulated data points, and is given by

$$
R^{2}=1-\frac{S S_{\text {res }}}{S S_{\text {tot }}}
$$

where $S S_{\text {res }}$ represents the sum of squares of residuals, expressed as 


$$
S S_{\text {res }}=\sum_{i=1}^{n}\left(V_{o p t, i}-\widehat{V}_{o p t, i}\right)^{2},
$$

and $S S_{\text {tot }}$ represents the total sum of squares, expressed as

$$
S S_{t o t}=\sum_{i=1}^{n}\left(V_{o p t, i}-\bar{V}_{o p t}\right)^{2} .
$$

The variables $n, V_{\text {opt }}$, and $\widehat{V}_{\text {opt }}$ in Eqs. (25) and (26) represent the sample size, the optimal hot water storage tank volume determined through simulation, and the optimal hot water storage tank volume predicted by the regression model, respectively. The variable $\bar{V}_{\text {opt }}$ is defined as the mean of $V_{\text {opt }}$ over the selected sample size, $n$, and is given by

$$
\bar{V}_{o p t}=\frac{1}{n} \sum_{i=1}^{n} V_{o p t, i} .
$$

The second performance metric is the relative model error, $e_{r e l}$, expressed as

$$
e_{\text {rel }}=\frac{\hat{V}_{o p t . i}-V_{o p t, i}}{V_{o p t, i}} .
$$

This metric is calculated for each individual residual across all scenarios. Results for any given scenario are plotted as a probability distribution in order to assess the quality of the model's fit. In general, it is desirable to have a probability distribution of relative model errors with a mean (accuracy) and standard deviation (precision) as close to zero as possible.

\subsection{Impact of noise on residuals}

The effect of random noise on the regression models is examined by testing cases with varying levels of relative noise. Normally distributed noise is added to each residual, with 
a mean of zero and a standard deviation, $\sigma_{\text {noise }}$, equivalent to a fraction of the highest amplitude contained in the residual, as shown by

$$
\sigma_{\text {noise }}=\sigma_{\text {rel }} \max \{\vec{a}\},
$$

where $\sigma_{r e l}$ is the relative noise level that varies between 0 and 1 , and $\vec{a}$ is the vector of amplitudes for the given residual.

In regression analysis, noise tends to introduce bias in the estimation of model parameters [13]. This is demonstrated in Figure 8.1 which shows four probability distributions of the relative model error for a given scenario subject to varying noise levels during both model fitting and testing. Figure 8.1a shows the distribution of a model fit to noiseless data (i.e. $\left.\sigma_{r e l}=0\right)$ in blue. This model is subsequently used to predict $\widehat{V}_{\text {opt }}$ for a set of residuals in which $\sigma_{r e l}=0.2$. The relative model error distribution corresponding to these predicted values is shown in orange, and is biased towards underprediction, as the distribution becomes broader. Figure $8.1 \mathrm{~b}$ shows the opposite effect, where the model is fit to data in which $\sigma_{\text {rel }}=0.2$, and tested on noiseless residuals. As in Figure 8.1a, bias is introduced into the model because the noise in the residuals differs from the noise for which the model was initially fit. 
(a)

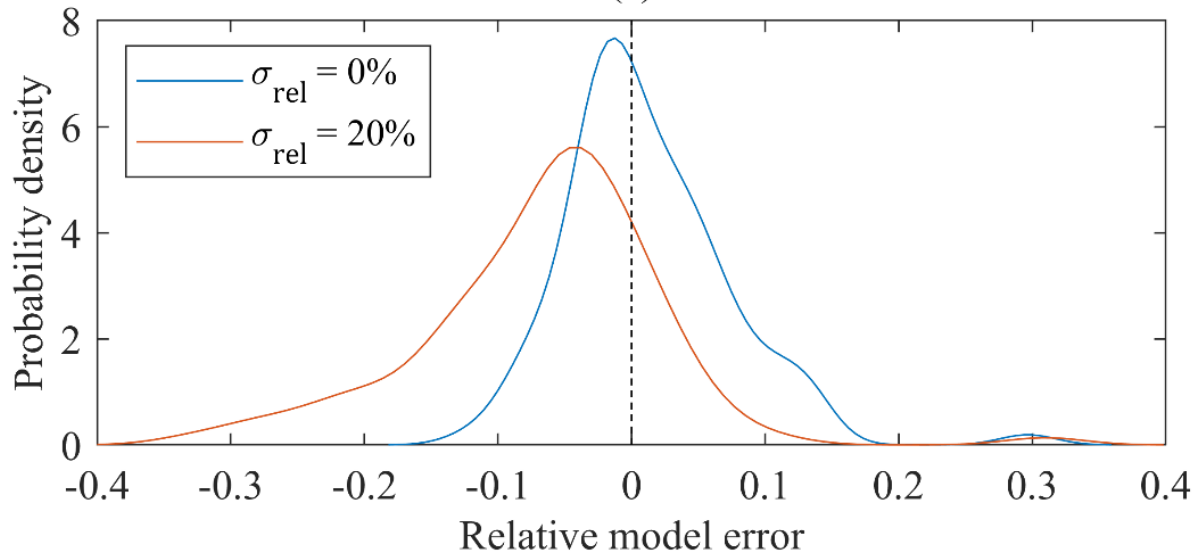

(b)

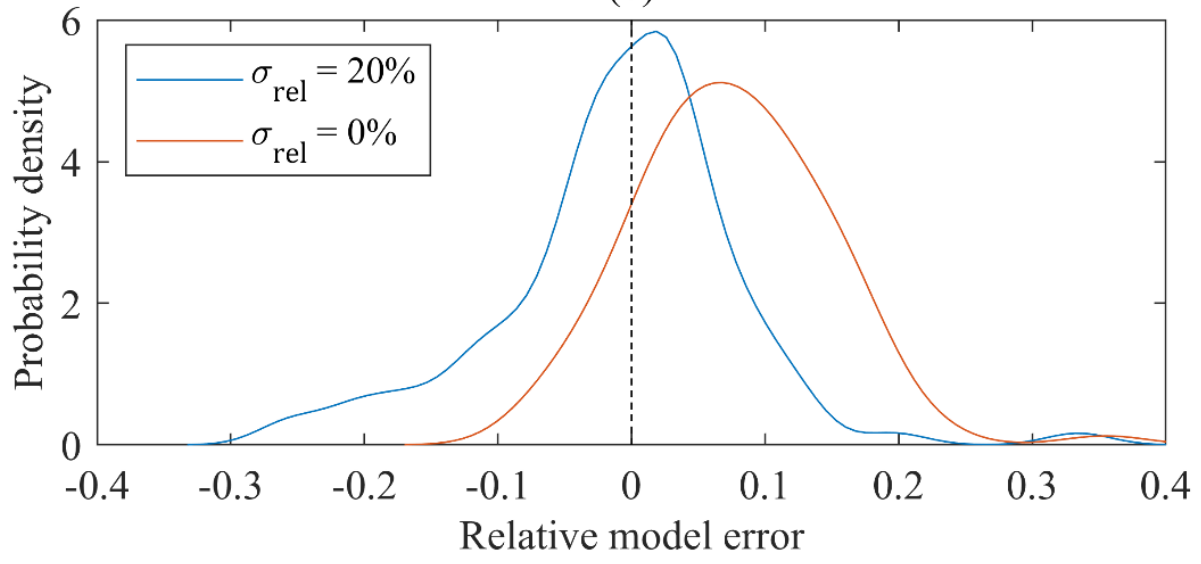

Figure 8.1: Probability distributions of the relative model error for a given scenario subject to varying noise levels during both model fitting (blue) and testing (orange). Bias is introduced into the model in both (a) where the model is fit to noiseless data, and (b) where the model is fit to data where $\sigma_{r e l}=0.2$.

In real systems residual signals are often noisy, however the level of noise is likely to vary considerably from one system to another. In the current study all residuals used to perform the regression analysis are generated according to Eq. (29) using a fixed value of $\sigma_{\text {rel }}=$ 0.15 . 


\section{Limiting cases of the regression model}

Prior to identifying the final regression model form (i.e. Eq. (23)), tests were conducted for several simplified cases. The first test case consisted of residuals containing a single sinusoid and no random noise $\left(n=1\right.$ and $\left.\sigma_{\text {noise }}=0\right)$. When only a single sinusoid is present, Eq. (23) simplifies to Eq. (22). Figure 9.1 shows a sample of optimization results for such residuals plotted against the sinusoid amplitude, where the marker shapes represent the period. The figure shows that for a given period, $V_{o p t}$ is linearly related to amplitude. The fact that the lines at different period are non-parallel also suggest an "interaction" term between phase and amplitude, where variables are said to interact in a regression model if they are multiplied together [13]. Referring to Eq. (22), if the period is held constant then $V_{o p t}$ is expected to be proportional to amplitude. Eq. (22) also contains the expected interaction term because the expression includes the product of amplitude and period. Both findings support the selected model represented by Eq. (23). 


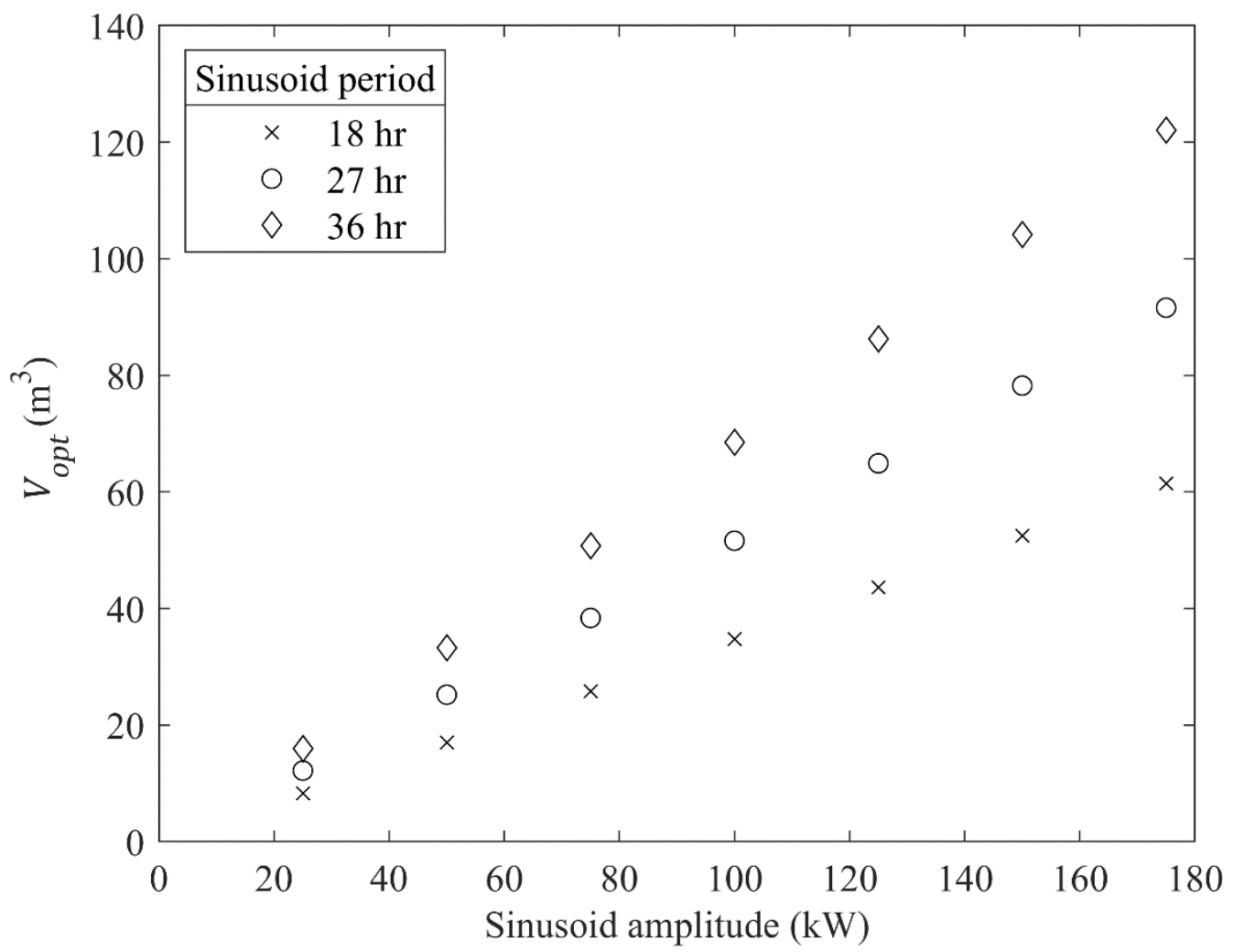

Figure 9.1: Optimal storage size plotted as a function of sinusoid amplitude and period for residuals with $n=1$.

When increasing the complexity of the test cases to higher values of $n$, the following observations were made:

- For the simple test cases with $n=2$ the relative model errors were biased towards overprediction of $V_{\text {opt }}$.

- When inspecting the residuals where the predicted volume was significantly larger than the optimal volume (i.e. $\widehat{V}_{\text {opt }} \gg V_{\text {opt }}$ ), it was observed that they were all cases where the two periods were integer multiples of each other, meaning that the sinusoids were harmonic. 
- In other test cases where $n \geq 3$, or random noise was added to the residual $\left(\sigma_{\text {rel }}>\right.$ 0), large overpredictions for residuals containing harmonics were not observed. These observations suggested that the model in Eq. (23) was applicable for all cases except when $n=2$ and $\sigma_{\text {noise }}=0$. While this is an unlikely scenario in a real system, the harmonic interaction occurring in the limiting case of $n=2$ was investigated further.

The overpredictions occurring for residuals with harmonic periods were corrected by adding several parameters, $b_{k}$, to the model, each being raised to a new predictor variable, $x_{k}$, which resulted in the model form

$$
\widehat{V}_{o p t}=\left(\sum_{i=1}^{2} c_{i} a_{i} p_{i}\right) b_{1}^{x_{1}} b_{2}^{x_{2}} b_{3}^{x_{3}} b_{4}^{x_{4}} .
$$

The values of $b_{k}$ were assigned through the parameter estimation process, since they are model parameters. They are non-linear parameters however, so OLS could not be directly used. This can be easily overcome with a logarithmic transformation to effectively linearize the parameters [13]. Because their purpose was to reduce the volume estimate in harmonic cases, their values were in the range of $0-1$. The value of $k$ ranged from 1-4, with each $b_{k} / x_{k}$ pair corresponding to a different harmonic multiple, $h$. Adopting the notation whereby the two sinusoids are labelled 1 and $2, h$ was defined by

$$
p_{2}=h p_{1} \quad\{h \in \mathbb{N} \mid h \geq 2\} \quad p_{1}<p_{2}
$$

and represents the harmonic ratio of the two periods $p_{1}$ and $p_{2}$, with $p_{2}$ being the larger of the two.

The overprediction errors resulting from harmonics were observed to be dependent on the value of $h$, as well as two other parameters: the relative phase, $\phi_{r e l}$ and the relative amplitude, $a_{r e l}$. The relative phase of the sinusoids, $\phi_{r e l}$, is expressed as 


$$
\phi_{\text {rel }}=\bmod \left(\frac{\phi_{1}-\phi_{2}}{p_{1}}, 1\right) \quad p_{1}<p_{2}
$$

where $\phi_{1}$ and $\phi_{2}$ are the phases of the two sinusoids, and the relative amplitude of the two sinusoids, $a_{r e l}$, is expressed as

$$
a_{r e l}=\frac{a_{2}}{a_{1}} .
$$

where $a_{1}$ and $a_{2}$ are their amplitudes.

Figure 9.2a presents a sample of relative model errors for sinusoids with $h=2$ as a function of both $\phi_{r e l}$ and $a_{r e l}$, while Figure $9.2 \mathrm{~b}$ does the same for $h=3$. These figures suggest that the overprediction error is much more strongly dependent on $\phi_{r e l}$ than on $a_{r e l}$. For example, all the lines of constant $a_{r e l}$ in Figure $9.2 \mathrm{~b}$ show a similar trend where overprediction errors are highest when $\phi_{\text {rel }}$ is close to 0.5 , and lowest when it is near 0 or 1. The difference in the lines for any given value of $\phi_{r e l}$ is relatively small. In contrast, the difference in overprediction error when changing $\phi_{r e l}$ for a given value of $a_{r e l}$ is significant. The value of $h$ appears to influence the pattern exhibited by the curves. Figure 9.2a shows that when $h=2$ there is a significant overprediction error at $\phi_{r e l}=0$, whereas it was seen that when $h=3$ in Figure $9.2 \mathrm{~b}$ that $\phi_{r e l}=0$ results in low overprediction errors. 

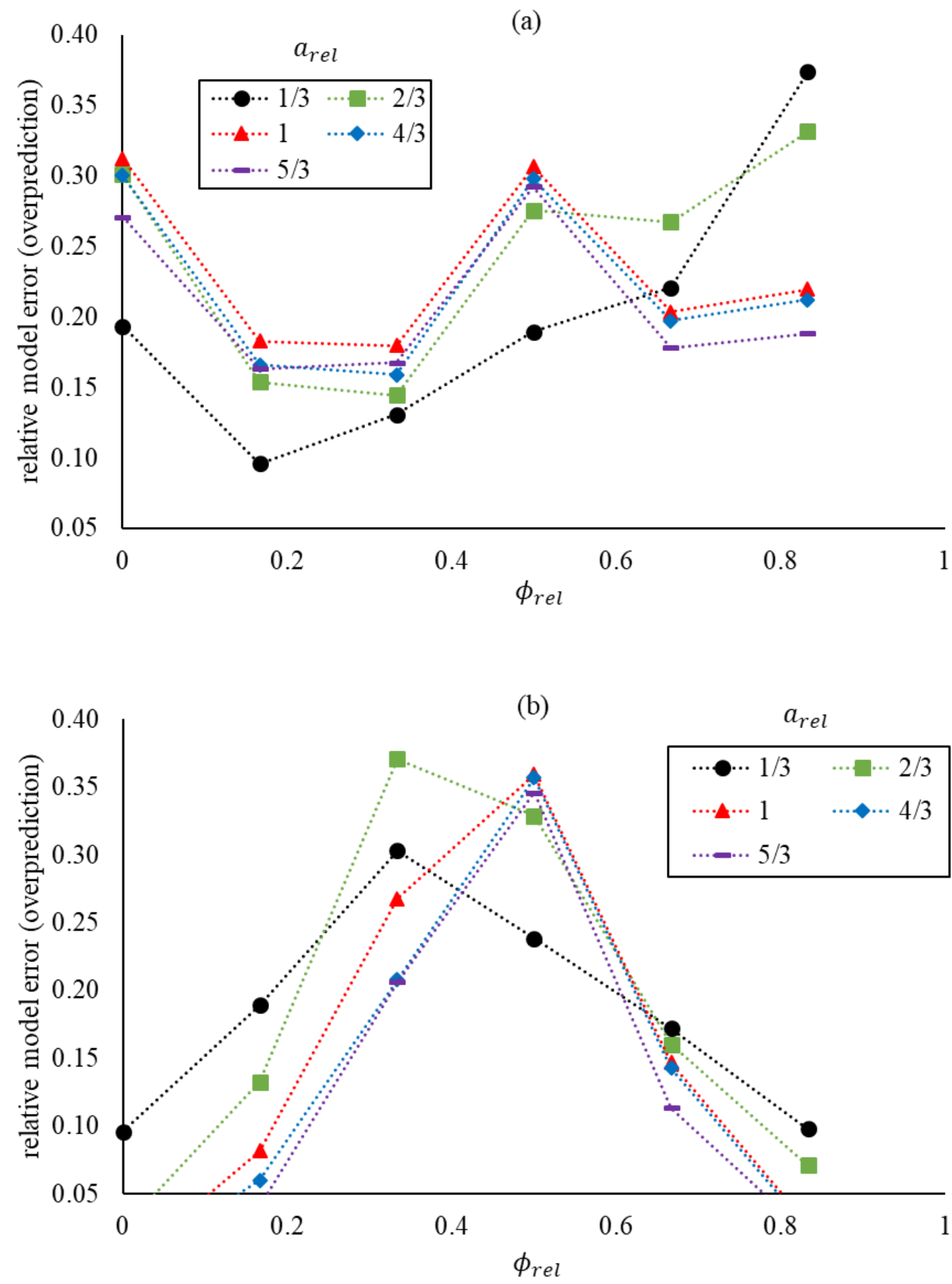

Figure 9.2: Relative model error as a function of $h, \phi_{r e l}$ and $a_{\text {rel }}$. Plots (a) and (b) represent sample relative model errors for cases with $h=2$ and $h=3$ respectively. 
The model in Eq (30) is influenced by $h, \phi_{r e l}$ and $a_{r e l}$ through the $x_{k}$ predictor variables, which are assigned for any residual as follows:

1. Based on the value of $h$ for the two sinusoids, the corresponding value of $k$ is selected in Table 9.1. This is represented as $k_{p}$.

2. The corresponding value of $x_{k_{p}}$ is assigned a value in the range of $0-1$, as a function of $\phi_{r e l}$ and $a_{r e l}$. This in effect applies the parameter $b_{k_{p}}$ to the model, to a degree determined by the value of $x_{k_{p}}$.

3. All other $x_{k}$ variables are assigned a value of 0 , effectively removing the corresponding $b_{k}$ parameters from the model.

Table 9.1: Index for selecting the value of $k_{p}$ for a residual with two sinusoids.

\begin{tabular}{cc}
\hline $\begin{array}{c}\text { Type of harmonic relationship } \\
\text { between the two sinusoids }\end{array}$ & Value of $k_{p}$ \\
\hline Not harmonic, or $h \geq 5$ & 1 \\
$h=2$ & 2 \\
$h=3$ & 3 \\
$h=4$ & 4 \\
\hline
\end{tabular}

This presented the problem of identifying the relationship between $x_{k}$ and the parameters $\phi_{r e l}$ and $a_{r e l}$. Rather than performing another regression analysis to develop a model, a large set of results like those presented in Figure 9.2 were collected and averaged to generate lookup tables for $x_{k}$. Combinations of $\phi_{r e l}$ and $a_{r e l}$ where the overprediction error was close to the maximum value (for example, the maximum overprediction is about 0.37 or $37 \%$ in Figure $9.2 \mathrm{~b}$ ) would be assigned $x_{k}=1$ to provide a larger reduction in predicted volume (recall that $b_{k}$ is in the range $0-1$ so a larger exponent $x_{k}$ results in a smaller volume estimate). By the same reasoning, combinations of $\phi_{r e l}$ and $a_{r e l}$ where the 
overprediction error was close to zero were assigned values of $x_{k}=0$, since no reduction in volume would be required. Finally, combinations of $\phi_{r e l}$ and $a_{r e l}$ resulting in intermediate overprediction errors were assigned values somewhere between $0-1$. These sampled results were then used to linearly interpolate values of $x_{k}$ for any given residual as required. This approach, while somewhat crude, provided reasonable results without adding too much additional complexity to the analysis.

Figure 9.3 shows the effect of using the modified model (i.e. Eq. (30)) which considers harmonics for residuals with two sinusoids. The blue line shows the distribution of relative errors resulting from fitting Eq. (23) to a data set of 624 random residuals; the tail on the far right of the distribution results from ignoring the effect of harmonic periods. The orange line shows the same when Eq. (30) is used instead; the model becomes more symmetric and more tightly centered around a mean of zero. This result shows that the effect of harmonics in cases of a residual with two sinusoids and little noise can be overcome by increasing the complexity of the model, however this approach is not practical. It is always an option to add more parameters to a model to increase its flexibility and performance; however, even if all the added parameters are shown to be significant (through a t-test), the risk of model overfitting increases. Overfitting occurs when the model attempts to fit to the noise present in the data, and generally reduces the model's predictive capability [13]. Because most systems encountered in practice will have residuals composed of more than two sinusoids, as well as a significant amount of random noise, the model described by Eq. (23) is considered the better choice for this method. For this reason, it is used to generate all the results in this thesis. 


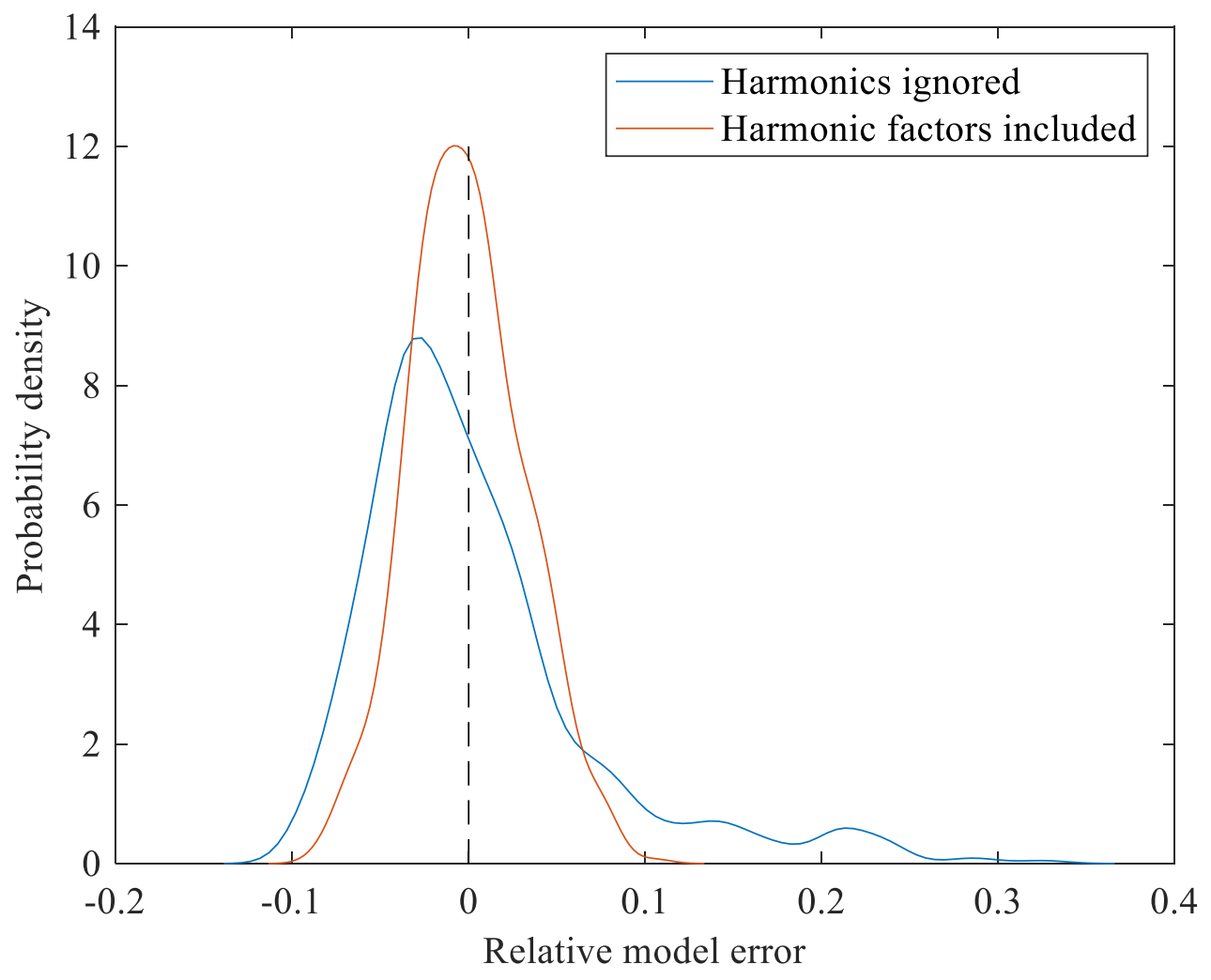

Figure 9.3: Probability distributions of relative model error for sinusoids with $n=2$. The blue distribution represents the relative model errors when harmonics are ignored (using Eq. (23)), while the orange distribution represents the relative model errors when additional model parameters are used to reduce the overprediction bias (using Eq. (30)). 


\section{Demonstration of sizing method via case study}

\subsection{Case study description}

A case study solar thermal energy conversion system designed for meeting the domestic hot water needs of a large hypothetical multi-unit residential building located in the city of Montréal, Canada, is modeled in TRNSYS to demonstrate the proposed hot water storage tank sizing method in practice. The system model, depicted in Figure 10.1, is similar to the model described in Chapter 4 in all respects except for the following:

- A solar thermal collector array with a total collector area of $1500 \mathrm{~m}^{2}$ is used as the primary heat source. The collector model is based on a high-performance flat-plate collector design described by Rockenbaugh et al. [36]. Due to the prevalence of extreme cold winter temperatures at this location, the solar thermal loop uses a $50 \%$ by weight propylene glycol working fluid [37] to prevent freezing, and is decoupled from the storage tank via a primary heat exchanger, $\mathrm{HX}_{1}$;

- $\mathrm{VSP}_{1}$ is always turned on when solar radiation is available. $\mathrm{VSP}_{2}$ is additionally turned on when the desired source temperature, $T_{6}$, can be achieved;

- A bypass loop, Bypass 1 , is added to the source loop as a hot water tempering mechanism;

- The cooling tower is connected to the source loop and operates using a predictive control strategy based on hourly solar irradiance data. At times of low irradiance, the cooling tower is activated when $T_{2}(t)$ exceeds $80^{\circ} \mathrm{C}$, and at times of high irradiance, the cooling tower is activated when $T_{2}(t)$ exceeds $65^{\circ} \mathrm{C}$. This strategy 
greatly reduces overheating of the working fluid, and minimizes the amount of heat wasted by the cooling tower;

- The storage tank has two inlets and two outlets;

- A second heat exchanger, $\mathrm{HX}_{2}$, is added on the load side to simulate aggregated consumer side heat exchangers;

- Heat exchangers operate adiabatically with a fixed approach temperature of $2.5^{\circ} \mathrm{C}$;

- A simulation time-step of 0.125 hours is used.

Fixed model inputs include the hot water storage tank volume, the solar collector array delivery temperature, the source temperature, and the load temperature, $T_{4}$. The source and load temperatures are assumed to be $95^{\circ} \mathrm{C}$ and $60^{\circ} \mathrm{C}$, respectively. Variable model inputs include the aggregated domestic hot water load, $Q_{\text {load }}(t)$, the solar thermal source, $Q_{\text {source }}(t)$, and the outdoor environmental temperature, $T_{\text {env }}(t)$. All other variables depicted in Figure 10.1 are output by the model. The aggregated domestic hot water load is estimated based on a study by Edwards et al. [22] in which annual domestic hot water load profiles are measured for 73 homes located in Québec, Canada in 2007. In the current study, these load profiles are aggregated, and a scaling factor is employed to increase the mean annual load to $100 \mathrm{~kW}$, a value that corresponds to the average domestic hot water load of approximately 225 homes. Each home is assumed to have a constant mains water temperature of $10^{\circ} \mathrm{C}$, and draw hot water at an average temperature of $57.5^{\circ} \mathrm{C}$. The solar thermal source is calculated using annual location-based weather data for Montréal, Canada, and scaled up (through selecting the collector area) such that the mean annual thermal energy recovered from the solar collector array is equivalent to the mean annual domestic hot water load (i.e. $100 \mathrm{~kW})$. 


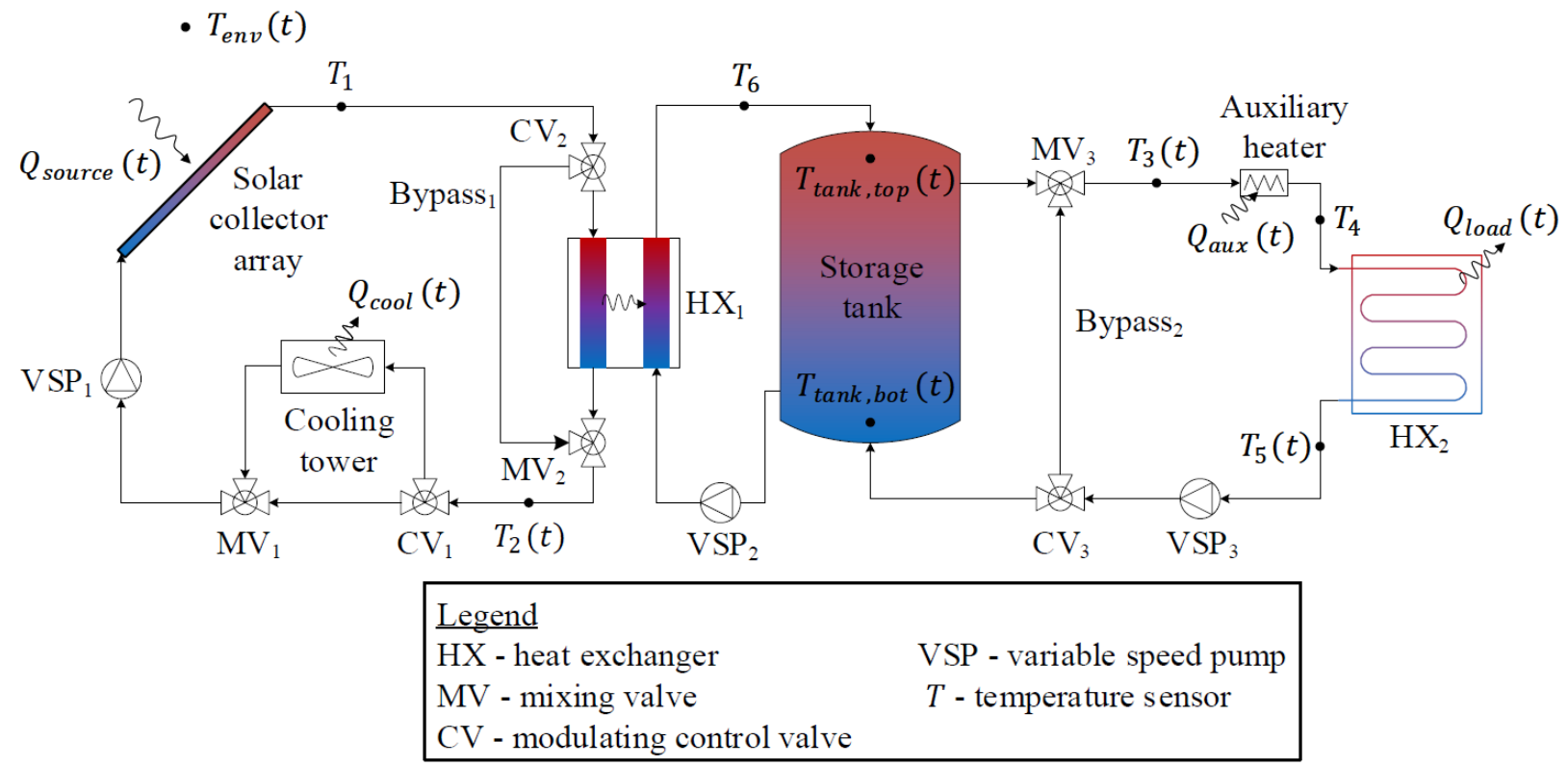

Figure 10.1: Schematic of transient numerical model developed to demonstrate the proposed sizing method on a case study solar thermal domestic hot water system located in Montréal, Canada.

\subsection{Case study configurations}

Six case study configurations, shown as A-F in Table 10.1, are developed that represent different combinations of the extraneous parameters described in Table 6.1. Both the tank environment, and the auxiliary energy price are varied, whereas the source and load temperatures are kept constant at $95^{\circ} \mathrm{C}$, and $60^{\circ} \mathrm{C}$, respectively. These temperatures are in line with those utilized in the case study simulation model. The auxiliary energy costs also match those used in the case study; at the time of writing the residential electricity rates provided by Hydro Québec are closest to the $0.07 \mathrm{USD} / \mathrm{kWh}$ rate (Rate D for residential customers is $0.0938 \mathrm{CAD} / \mathrm{kWh}$, or about $0.0713 \mathrm{USD} / \mathrm{kWh}$ ) [38]. 
Table 10.1: Case study configurations based on varying combinations of extraneous parameters

\begin{tabular}{lllll}
\hline $\begin{array}{l}\text { Case study } \\
\text { configuration }\end{array}$ & $\begin{array}{l}\text { Tank } \\
\text { environment }\end{array}$ & $\begin{array}{l}\text { Source } \\
\text { temperature }\left({ }^{\circ} \mathrm{C}\right)\end{array}$ & $\begin{array}{l}\text { Load temperature } \\
\left({ }^{\circ} \mathrm{C}\right)\end{array}$ & $\begin{array}{l}\text { Auxiliary } \\
\text { energy price } \\
(\mathrm{USD} / \mathrm{kWh})\end{array}$ \\
\hline A & outdoor $($ cold) & 95 & 60 & 0.13 \\
$\mathrm{~B}$ & outdoor (cold) & 95 & 60 & 0.105 \\
$\mathrm{C}$ & outdoor (cold) & 95 & 60 & 0.07 \\
$\mathrm{D}$ & indoor & 95 & 60 & 0.13 \\
$\mathrm{E}$ & indoor & 95 & 60 & 0.105 \\
$\mathrm{~F}$ & indoor & 95 & 60 & 0.07 \\
\hline
\end{tabular}

Each case study configuration in Table 10.1 corresponds to one of the 36 optimization scenarios described in Chapter 6. To predict the optimal hot water storage tank volume, $\widehat{V}_{o p t}$, for each configuration, $c, a$, and $p$ parameter sets need to be determined and input into Eq. (23). The first of these parameter sets (i.e. c parameters) are obtained from the results of the regression analysis of the corresponding optimization scenario. The latter two parameter sets (i.e. $a$ and $p$ parameters) are determined from the periodogram of the system's residual heating profile which is obtained by applying a FFT waveform conversion.

To demonstrate the performance of the proposed hot water storage tank sizing method, $\widehat{V}_{\text {opt }}$ values for each case study configuration are compared with corresponding $V_{\text {opt }}$ values obtained via simulation. The associated $N P C$ values for each configuration are also shown for comparison. 


\section{Results and discussion}

\subsection{Regression model parameters for optimization scenarios}

The results of the regression analysis for the 36 optimization scenarios considered in this study are presented in Table 11.1 below. For each scenario, both the regression model parameters $\left(c_{1}-c_{5}\right)$, and the coefficient of determination, $R^{2}$, are shown. The most influential extraneous variables to model $\mathrm{R}^{2}$ are the source and load temperatures of the scenario, and to a lesser extent, the auxiliary energy price. The tank environment plays a relatively minor role in the variability of $\mathrm{R}^{2}$ in the models. The average $R^{2}$ value for all scenarios with $95^{\circ} \mathrm{C}$ source temperature and $60^{\circ} \mathrm{C}$ load temperature $(95 / 60)$ is 0.966 , indicating a strong model fit, whereas the $75 / 60$ scenarios have an average $R^{2}$ of 0.625 , with individual values varying significantly between 0.342 and 0.822 , suggesting weaker and less consistent model fits. The 95/40 and 75/40 scenarios, on the other hand, show intermediate results with average $R^{2}$ of 0.864 and 0.814 , respectively. These trends can be partly explained by considering the system objective function: a univariate function relating net present cost to tank volume. Scenarios with the lowest $R^{2}$ values are characterized by objective functions with broader and less distinct optima, and tend to be those in which 1) small differences exist between source and load temperatures, and 2) auxiliary energy prices are lowest. Having a relatively small difference between source and load temperature causes the objective function to become broader near the optimum as each marginal unit of volume has an effectively smaller useful energy storage capacity. Similarly, as the auxiliary energy price decreases, the objective function becomes broader as incremental energy savings or losses from changing the tank volume become less 
valuable. The broader the objective function is near the optimum the more likely the solver is to be influenced by "jagged" behaviour and end up selecting a local optimum; however, a broader objective function also means that the penalty to net present cost for being further from the optimum tank volume is lower. Thus, while the $R^{2}$ values may be lower in the $75 / 60$ scenarios, results may still be relied upon to a considerable degree because most of the cost savings are obtained simply by being close to the optimum. In general, the proposed method provides the most accurate volume estimates for scenarios in which the difference between source and load temperatures is $35^{\circ} \mathrm{C}$ or greater (particularly when the source temperature is $95^{\circ} \mathrm{C}$ ), and where auxiliary energy prices exceed $0.105 \mathrm{USD} / \mathrm{kWh}$.

Figure 11.1 shows the relative model error, $e_{r e l}$, probability distributions, depicted as box-and-whisker plots, for each of the optimization scenarios. These plots show the spread in error associated with each scenario, where the central solid mark indicates the distribution median, the top and bottom solids marks indicate the first and third quartiles, respectively, and the dashed "whiskers" extend to the minimum and maximum error values that are not deemed to be outliers. Outliers are marked in red and are so labelled when values are more than 1.5 times the value of the interquartile range away from either the first or third quartile. In a similar pattern to the $R^{2}$ results, the $95 / 60$ scenarios (Figure 11.1a) are the best performers with distributions centered tightly around zero. The $75 / 60$ scenarios (Figure 11.1c), on the other hand, have very wide error distributions ranging from about -0.5 to 0.5 , suggesting a poor model fit. The $75 / 40$ scenarios (Figure 11.1d) also show relatively poor performance compared to the scenarios with 95 source temperature, however they differ from the $75 / 60$ scenarios in that they are not quite as widely distributed but have a larger number of outliers. 
Table 11.1: Regression model parameters $\left(c_{1}-c_{5}\right)$ and corresponding $R^{2}$ value as a function of hot water storage tank source and load temperatures, tank environment, and auxiliary energy price for 36 optimization scenarios.

\begin{tabular}{|c|c|c|c|c|c|c|c|c|}
\hline \multirow{2}{*}{$\begin{array}{l}\text { Source/load } \\
\text { temperature } \\
\qquad\left({ }^{\circ} \mathrm{C}\right)\end{array}$} & \multirow{2}{*}{$\begin{array}{c}\text { Tank } \\
\text { environment }\end{array}$} & \multirow{2}{*}{$\begin{array}{c}\text { Auxiliary } \\
\text { energy price } \\
\text { (USD/kWh) }\end{array}$} & \multicolumn{5}{|c|}{$\begin{array}{l}\text { Regression model parameters } \\
\qquad\left(\times 10^{-2} \mathrm{~m}^{3} / \mathrm{kWh}\right)\end{array}$} & \multirow[t]{2}{*}{$R^{2}$} \\
\hline & & & $c_{1}$ & $c_{2}$ & $c_{3}$ & $c_{4}$ & $c_{5}$ & \\
\hline $95 / 60$ & indoor & 0.07 & 2.01 & 1.75 & 1.63 & 1.44 & 0.38 & 0.970 \\
\hline $95 / 60$ & indoor & 0.105 & 1.97 & 1.82 & 1.58 & 1.18 & 0.92 & 0.962 \\
\hline $95 / 60$ & indoor & 0.13 & 2.04 & 1.81 & 1.47 & 1.26 & 0.52 & 0.979 \\
\hline $95 / 60$ & outdoor (cold) & 0.07 & 1.94 & 1.79 & 1.48 & 1.11 & 0.99 & 0.961 \\
\hline $95 / 60$ & outdoor (cold) & 0.105 & 1.95 & 1.72 & 1.53 & 1.44 & 0.98 & 0.951 \\
\hline $95 / 60$ & outdoor (cold) & 0.13 & 2.00 & 1.62 & 1.74 & 1.25 & 0.63 & 0.951 \\
\hline $95 / 60$ & outdoor (warm) & 0.07 & 1.99 & 1.78 & 1.71 & 1.33 & 0.50 & 0.975 \\
\hline $95 / 60$ & outdoor (warm) & 0.105 & 1.96 & 1.81 & 1.54 & 1.24 & 0.86 & 0.964 \\
\hline $95 / 60$ & outdoor (warm) & 0.13 & 2.03 & 1.78 & 1.48 & 1.22 & 0.57 & 0.978 \\
\hline $95 / 40$ & indoor & 0.07 & 1.50 & 1.19 & 0.71 & 1.26 & 0 & 0.877 \\
\hline $95 / 40$ & indoor & 0.105 & 1.40 & 1.39 & 1.50 & 0 & 0 & 0.873 \\
\hline $95 / 40$ & indoor & 0.13 & 1.42 & 1.23 & 1.25 & 0.96 & 0 & 0.901 \\
\hline $95 / 40$ & outdoor (cold) & 0.07 & 1.42 & 1.05 & 0.96 & 1.12 & 0 & 0.880 \\
\hline $95 / 40$ & outdoor (cold) & 0.105 & 1.32 & 1.28 & 1.03 & 0.67 & 0 & 0.859 \\
\hline $95 / 40$ & outdoor (cold) & 0.13 & 1.35 & 1.16 & 1.03 & 0.84 & 0 & 0.885 \\
\hline $95 / 40$ & outdoor (warm) & 0.07 & 1.38 & 1.44 & 0.58 & 1.00 & 0.82 & 0.777 \\
\hline $95 / 40$ & outdoor (warm) & 0.105 & 1.32 & 1.42 & 1.09 & 0.97 & 0 & 0.872 \\
\hline $95 / 40$ & outdoor (warm) & 0.13 & 1.44 & 1.27 & 0.83 & 1.10 & 0 & 0.853 \\
\hline $75 / 60$ & indoor & 0.07 & 3.92 & 1.00 & 2.83 & 0 & 0 & 0.342 \\
\hline $75 / 60$ & indoor & 0.105 & 3.83 & 4.31 & 0 & 0 & 0 & 0.628 \\
\hline $75 / 60$ & indoor & 0.13 & 4.15 & 2.60 & 1.49 & 2.87 & 0 & 0.822 \\
\hline $75 / 60$ & outdoor (cold) & 0.07 & 3.95 & 2.18 & 0 & 0 & 0 & 0.552 \\
\hline $75 / 60$ & outdoor (cold) & 0.105 & 3.92 & 2.74 & 1.42 & 0 & 0 & 0.768 \\
\hline $75 / 60$ & outdoor (cold) & 0.13 & 3.88 & 2.80 & 1.51 & 2.01 & 0 & 0.722 \\
\hline $75 / 60$ & outdoor (warm) & 0.07 & 4.03 & 2.23 & 0 & 0 & 0 & 0.503 \\
\hline $75 / 60$ & outdoor (warm) & 0.105 & 3.75 & 3.70 & 1.31 & 0 & 0 & 0.540 \\
\hline $75 / 60$ & outdoor (warm) & 0.13 & 3.97 & 3.33 & 0.86 & 3.13 & 0 & 0.752 \\
\hline $75 / 40$ & indoor & 0.07 & 1.85 & 1.30 & 1.63 & 1.66 & 0 & 0.820 \\
\hline $75 / 40$ & indoor & 0.105 & 1.84 & 1.55 & 1.17 & 1.34 & 0 & 0.918 \\
\hline $75 / 40$ & indoor & 0.13 & 1.87 & 1.38 & 1.63 & 1.70 & 0 & 0.867 \\
\hline $75 / 40$ & outdoor (cold) & 0.07 & 1.74 & 1.50 & 1.41 & 1.99 & 0 & 0.743 \\
\hline $75 / 40$ & outdoor (cold) & 0.105 & 1.72 & 2.32 & 0.62 & 1.63 & 0 & 0.748 \\
\hline $75 / 40$ & outdoor (cold) & 0.13 & 1.80 & 2.14 & 0.62 & 2.37 & 0 & 0.765 \\
\hline $75 / 40$ & outdoor (warm) & 0.07 & 1.80 & 1.45 & 1.76 & 1.27 & 0 & 0.761 \\
\hline $75 / 40$ & outdoor (warm) & 0.105 & 1.79 & 1.61 & 1.32 & 1.32 & 0 & 0.891 \\
\hline $75 / 40$ & outdoor (warm) & 0.13 & 1.79 & 1.57 & 1.55 & 1.51 & 0 & 0.818 \\
\hline
\end{tabular}


(a)

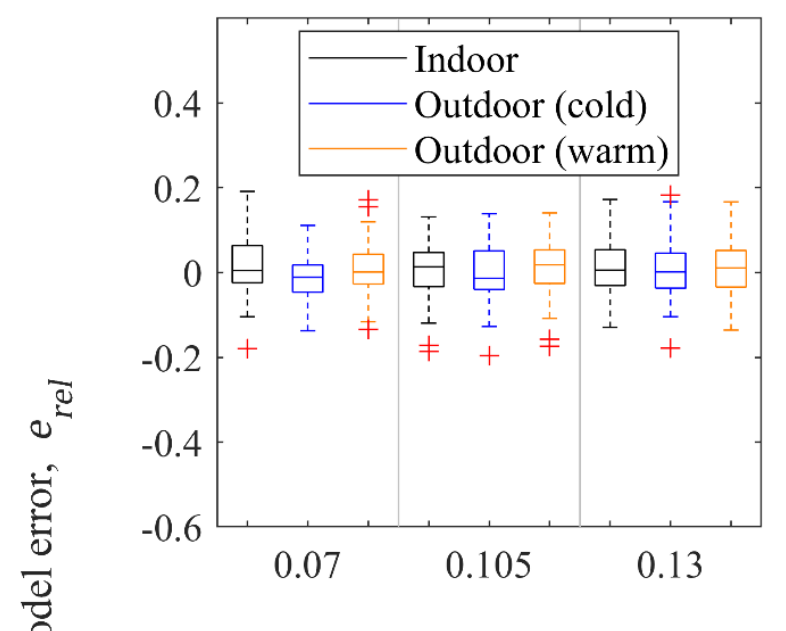

(c)

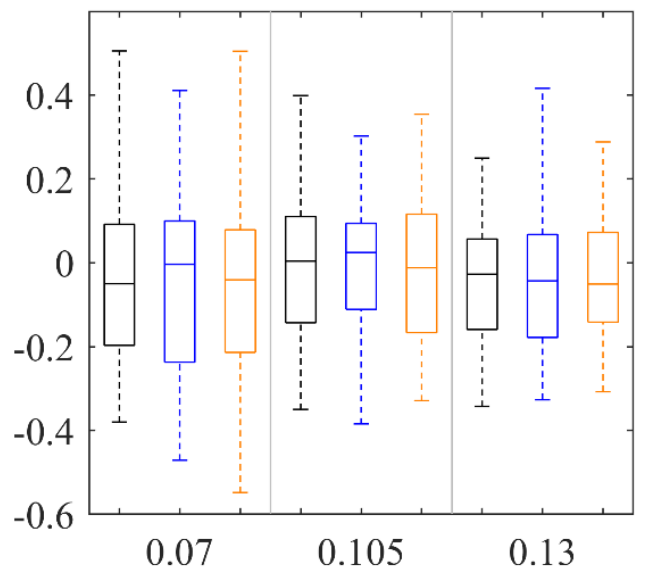

(b)

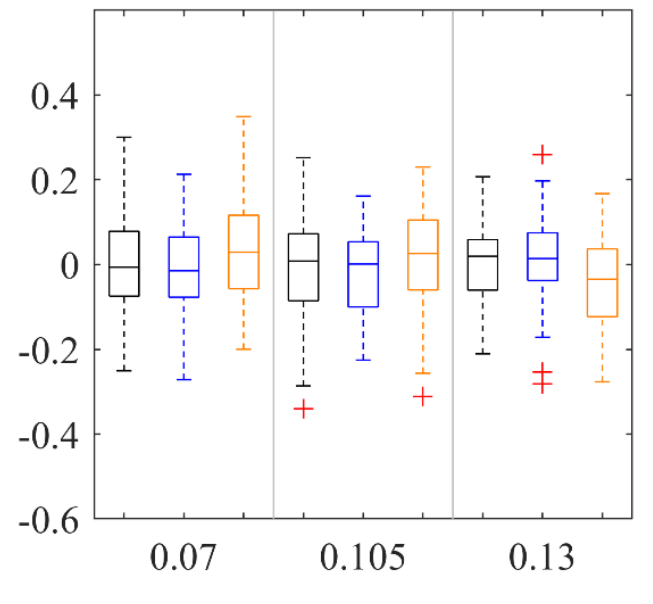

(d)

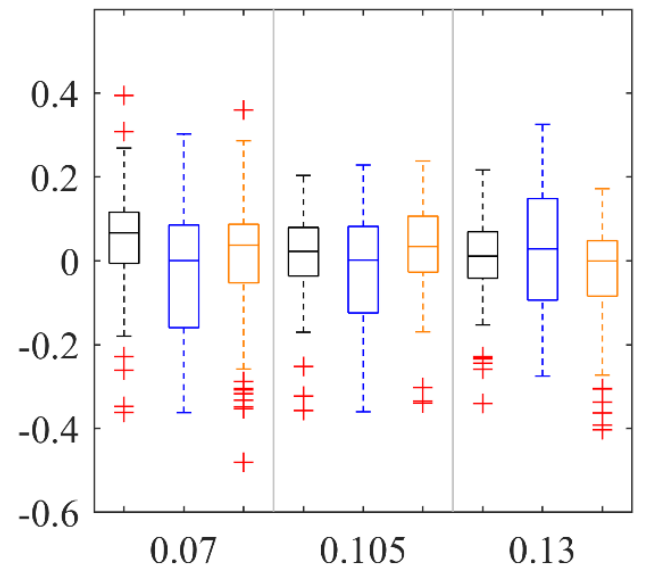

Auxiliary energy price (USD/kWh)

Figure 11.1: Box-and-whisker plots depicting relative model error, $e_{r e l}$, probability distributions for 36 optimization scenarios. (a), (b), (c), and (d) refer to scenarios with source and load temperatures of $95^{\circ} \mathrm{C}$ and $60^{\circ} \mathrm{C}, 95^{\circ} \mathrm{C}$ and $40^{\circ} \mathrm{C}, 75^{\circ} \mathrm{C}$ and $60^{\circ} \mathrm{C}$, and $75^{\circ} \mathrm{C}$ and $40^{\circ} \mathrm{C}$, respectively. 


\subsection{Application of regression models to case study}

\section{configurations}

Table 11.2 shows both the simulated and predicted values of the optimal hot water storage tank volume for all case study configurations presented in Chapter 10.2.

Table 11.2: Case study results by configuration. $V_{o p t}$, and $\widehat{V}_{o p t}$ represent the simulated and predicted optimal hot water storage tank volume, respectively; whereas $e_{r e l}$, and NPC represent the relative model error and system net present cost, respectively.

\begin{tabular}{lllllll}
\hline $\begin{array}{l}\text { Case study } \\
\text { configuration }\end{array}$ & $\begin{array}{l}V_{\text {opt }} \\
\left(\mathrm{m}^{3}\right)\end{array}$ & $\begin{array}{l}\hat{V}_{\text {opt }} \\
\left(\mathrm{m}^{3}\right)\end{array}$ & $e_{\text {rel }}$ & $\begin{array}{l}\text { NPC }- \\
\text { simulated } \\
(\text { MUSD) }\end{array}$ & $\begin{array}{l}\text { NPC - } \\
\text { predicted } \\
\text { (MUSD) }\end{array}$ & $\begin{array}{l}\text { NPC w/o } \\
\text { storage } \\
\text { (MUSD) }\end{array}$ \\
\hline A & 106 & 102.9 & -0.03 & 0.392 & 0.392 & 0.756 \\
B & 103 & 102.2 & -0.01 & 0.341 & 0.341 & 0.624 \\
C & 87 & 103.0 & 0.18 & 0.269 & 0.270 & 0.439 \\
D & 109 & 106.6 & -0.02 & 0.388 & 0.388 & 0.756 \\
E & 108 & 104.8 & -0.03 & 0.338 & 0.338 & 0.624 \\
F & 89 & 104.8 & 0.18 & 0.267 & 0.268 & 0.439 \\
\hline
\end{tabular}

To demonstrate the proposed sizing method in practice, Table 11.3 shows for case study configuration B the parameters needed to calculate $\widehat{V}_{\text {opt }}$ using Eq. (23). The $c$ parameters are obtained from Table 11.1, whereas the $a$ and $p$ parameters are obtained from the periodogram of the system's residual heating profile. Both the residual heating profile and corresponding periodogram are shown in Figure 11.2. Although the periodogram includes four significant peaks, the first one (i.e. the peak with a frequency roughly equal to zero) is ignored in the calculation as it corresponds to a yearly period, and as such is not relevant to diurnal storage. 
Table 11.3: Parameters used for calculating $\widehat{V}_{\text {opt }}$ for case study configuration B.

\begin{tabular}{cccc}
\hline$i$ & $\begin{array}{c}c_{i} \\
\left(\times 10^{-2} \mathrm{~m}^{3} / \mathrm{kWh}\right)\end{array}$ & $p_{i}(\mathrm{~h})$ & $\left.a_{i}(\mathrm{~kW})\right)$ \\
\hline 1 & 1.95 & 24 & 139.7 \\
2 & 1.72 & 12 & 149.5 \\
3 & 1.53 & 8 & 48.5 \\
4 & 1.44 & 0 & 0 \\
5 & 0.98 & 0 & 0 \\
\hline
\end{tabular}

(a)

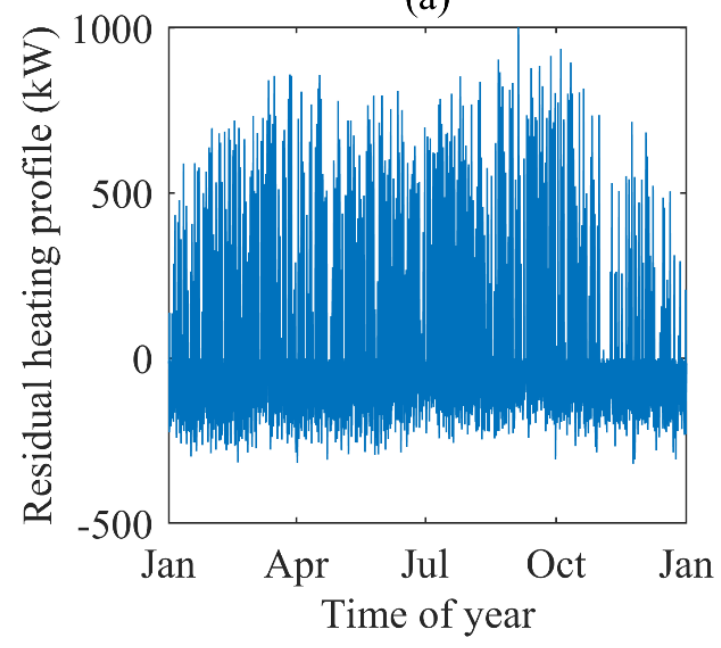

(b)

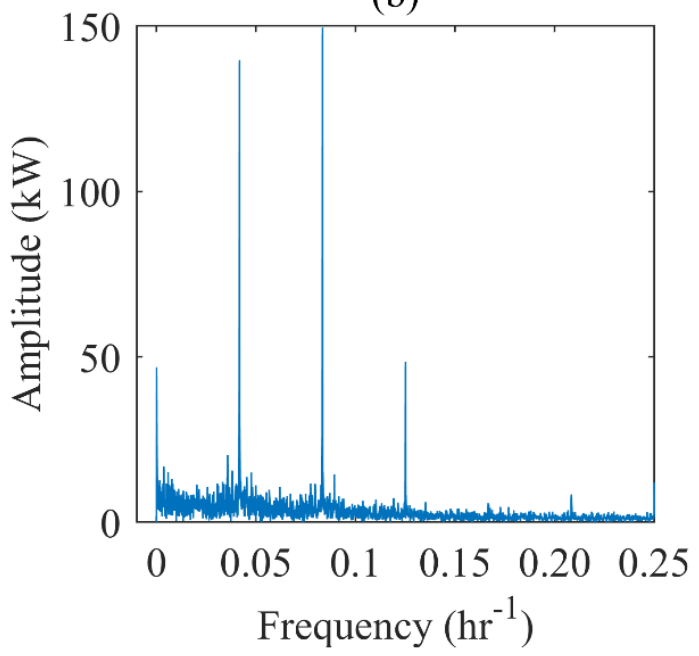

Figure 11.2: Annual residual heating profile (a), and corresponding periodogram (b) for case study configuration B.

Values of the relative model error and system net present cost, with and without (w/o) hot water storage, are also shown in Table 11.2 for each case study configuration. The relative model error is used to quantify the performance of the proposed hot water storage tank sizing method, and the net present cost is used to demonstrate the potential economic benefit from including storage capacity in the system. Error values corresponding to case study configurations $\mathrm{A}, \mathrm{B}, \mathrm{D}$, and $\mathrm{E}$ are lower than $3 \%$, indicating that simulated and predicted values for the optimal storage tank volume are in close agreement. This trend is illustrated in Figure 11.3 below which depicts the objective function for case study 
configuration B with nearly identical $V_{o p t}$, and $\widehat{V}_{o p t}$ values. It should be noted that the objective function in Figure 11.3 is very shallow near the objective function, that is, the NPC changes only slightly with volume near the optimum. This shallow slope is unique to the case study; in the simplified model used to generate the regression results there was generally a more distinct minimum with a steeper slope on either side. The shallow slope is attributed to the fact that the dynamics of the case study model are different than those from the simplified model, namely that in the case study system the residual is itself somewhat dependent on the storage tank volume: the temperature of the storage tank influences the temperatures of the antifreeze working fluid, which then in turn influences how much heat can be recovered at the solar collector. This finding supports the use of a simplified sizing method like the one developed here, as it suggests that one reaps most of the cost savings in such a system simply by being close to the optimum, meaning that there is some room for error in selecting the optimum storage size. This is also reassuring since in practice hot water storage tanks are generally not available in any size; even if a designer deviates somewhat from the optimal volume by selecting a ready-made hot water storage tank they can still obtain most of the cost savings.

Error values for configurations $\mathrm{C}$ and $\mathrm{F}$, on the other hand, are much greater at approximately $18 \%$. The main reason for these high error values is due to the low auxiliary energy price (\$0.07 USD/kWh) associated with these configurations. As the auxiliary energy price decreases, the objective function becomes broader near the optimum. Consequently, varying the storage volume in this broad region results in little to no change in the system NPC. This trend is shown in Table 11.2, where for each case study configuration, including those with high error values, system $N P C$ s corresponding to 
predicted storage volumes are nearly identical to those obtained from simulation. For all case study configurations, regardless of error, adding an optimally sized hot water storage tank to the system results in cost savings of between $38.5-48.7 \%$.

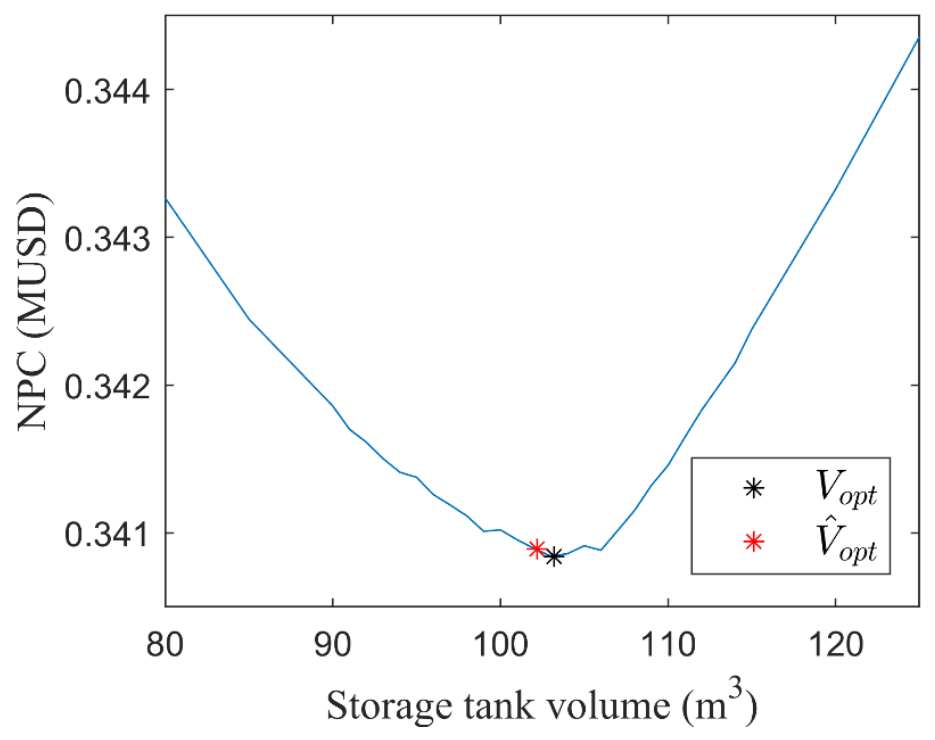

Figure 11.3: Objective function for case study configuration B showing values of $V_{\text {opt }}$, and $\widehat{V}_{\text {opt }}$ nearly overlapping.

\subsection{Sizing method applicability and unresolved issues}

Although the proposed hot water storage tank sizing method is highly versatile in that it can be applied to any arbitrarily complex system residual heating profile, it relies on several assumptions that may not reflect every user's circumstances. For example, fixed unit costs are assumed in the objective function, and fixed values of storage tank variables such as the height-to-diameter ratio, insulation thickness, and inlet height on the discharge loop, are assumed in the optimization scenarios. Furthermore, only a limited number of optimization scenarios are presented to deal with extraneous variables. Despite these restrictions, which are implemented to limit the computational intensity of the study, the 
method is still applicable to a wide range of conventional heating systems. Moreover, the tools required from a practitioner standpoint to utilize the proposed method are widely available at little to no cost. For instance, most data analysis tools including Microsoft Excel have either built-in or downloadable FFT capability.

A few unresolved issues remain concerning the proposed sizing method; these issues are outlined as follows:

1. For all optimization scenarios, it is assumed that the mean of the residual heating profile is equal to zero. While it is reasonable to assume that source and load profiles should have similar means in order to make the most effective use of the hot water storage tank, the effect of varying these means relative to each other has not been considered in this study.

2. There may be considerable uncertainty in the residual heating profile. For example, the residual can vary dramatically over time due to changing occupancy patterns for building-related loads. In order to obtain a more reliable estimate of $\widehat{V}_{o p t}$, it may be prudent for a practitioner to test for sensitivity by including multiple residual heating profiles in the analysis based on historical measured data. When several years of data are not available, statistical techniques can be used to create synthetic profiles [39].

3. The proposed sizing method is not suitable for systems whose residual heating profile consists of only two sinusoids (i.e. $n=2$ ). These cases result in a significant bias towards overprediction of the storage tank volume (i.e. high positive values of $e_{r e l}$ ) whenever the two sinusoids are harmonic. Sinusoids are said to be harmonic when the period of one sinusoid is a whole number multiple of the other 
sinusoid's period. This precludes the sizing method from being used on systems with a perfectly sinusoidal source paired with a perfectly sinusoidal load, or a source consisting of two sinusoids paired with a constant load. Fortunately, most systems are not so simple. Moreover, any interaction between harmonic sinusoids becomes negligible for cases where $n>2$, or $\sigma_{r e l}>0.05$.

4. The impacts of varying the noise level included in the residual heating profile on the response surface model results are unknown. To address this issue several additional optimization scenarios could be developed in which noise is introduced as an extraneous variable.

5. Many types of systems may have some dominant periods outside of the diurnal range. For example, solar thermal and hydro sources may exhibit significant seasonal variation, and wind energy sources may have significant sub-hourly variation. How these influence the optimum volume for a diurnal storage, remains to be investigated. 


\section{Summary of contributions}

This thesis provides the following contributions to the body of research on hot water storage:

1. The development of a novel methodology to quickly determine the volume for a hot water thermal storage tank that provides nearly optimal economic performance. The method is built upon well-established analysis techniques and only requires the user to provide the system residual profile, operating temperatures, auxiliary energy price, and climate type. Through its simplicity the method facilitates the incorporation of hot water storage tanks into existing systems with intermittent sources and loads, thereby supporting the uptake of clean energy technologies like renewables and waste heat recovery.

2. A verification of the hot water storage tank sizing method via a case study of a large residential building in Montréal, Canada.

3. A discussion of the ways in which the proposed methodology could be refined or extended to provide more robust sizing estimates for a wider range of systems. Currently the sizing method is best applied to hot water storage systems with large auxiliary energy costs and large differences between source and load temperatures.

The work completed in this thesis has been submitted to the international peer reviewed journal Applied Energy (see reference below) and is currently under review.

- D. Stalinski and J. Duquette, "Development of a simplified method for optimally sizing hot water storage tanks subject to short-term intermittent charge/discharge cycles," Applied Energy [under review]. 


\section{Conclusions and future work}

Optimal sizing of hot water storage capacity is an important consideration for reducing costs and increasing overall efficiency in many conventional heating systems. A simplified analytical method for optimally sizing hot water storage tanks based on a system's residual heating profile was presented in this study. A numerical hot water storage tank model was developed in TRNSYS to represent an arbitrary system subject to short-term intermittent charge and discharge cycles. Simulations were run to determine the optimal hot water storage tank volume for a large set of randomly generated residual heating profiles under 36 distinct modelling scenarios that encompass different combinations of the following four extraneous variables: heat source temperature, load service temperature, auxiliary energy price and tank environment temperature. The residual heating profiles were decomposed using a FFT algorithm, and mathematical models were developed using OLS regression to relate the profiles' amplitudes and periods to the optimal hot water storage tank volume. Results show that all scenarios with a source temperature of $95^{\circ} \mathrm{C}$, a source to load temperature difference of at least $35{ }^{\circ} \mathrm{C}$, and an auxiliary energy price of at least $0.105 \mathrm{USD} / \mathrm{kWh}$ have associated $\mathrm{R}^{2}$ values of 0.8 or greater. This finding indicates that under the stated conditions, the proposed sizing method is able to predict the optimal storage tank volume with a high degree of confidence. A case study was used to test the method for a hypothetical solar-thermal DHW system in Montréal, Canada. The sizing method produced near optimal results for all simulations in which the auxiliary energy price was at least $0.105 \mathrm{USD} / \mathrm{kWh}$, however errors of roughly $18 \%$ were seen for cases in which the auxiliary energy price was $0.07 \mathrm{USD} / \mathrm{kWh}$. 
With regards to future work, a wider range of variables should be considered to widen the range of applicability of the proposed sizing method. One of the most important variables in this respect is the hot water storage tank operating strategy. In the current study, a simple storage operating strategy is utilized that is based on maximizing resource efficiency. Several other operating strategies such as those utilized for electrical grid load shifting and/or peak shaving can also be investigated [40]. As the operating strategy would be treated as a decision variable to be co-optimized alongside storage size, multivariate optimization approaches would be required which would significantly increase computational intensity. For such instances, variable auxiliary energy pricing structures can be introduced instead of using a fixed auxiliary energy price. Since the pricing structure is generally an extraneous variable outside of the designer's control, this would amount to adding more scenarios, which would also increase computational requirements.

Assuming the availability of more computational resources, there is also a need to perform a detailed sensitivity analysis, particularly for the assumed parameters in the objective function of the optimization given by Eq. (16). As these assumed costs and parameters may change from year to year, these models would likewise need to be periodically updated to maintain their accuracy. Additionally, a larger number of residuals could be used for each scenario to improve the model-fitting process.

The method was based on a stratified hot water storage tank, where the stratification was maintained through the density-temperature relationship for water, as well as by selecting the inlets and outlets for the storage tank accordingly. The model did not consider how additional enhancements to stratification through physical mechanisms, like baffles, 
could impact the model results. Future work could examine the application of this method for other configurations of hot water storage tanks.

Despite all of these opportunities for refinement, the proposed sizing method is a promising alternative for optimally sizing several typical hot water storage tank configurations, particularly due to its speed and ease of application. The suggested future work would help to refine the method and make it more broadly applicable to a wider and more complex range of systems. Furthermore, this same methodology could be extended to other forms of TES systems like latent and thermochemical. 


\section{References}

[1] Natural Resources Canada, "Energy Factbook 2019-2020,” 2019.

[2] ASHRAE, “ANSI/ASHRAE/IES Standard 90.1-2019 - Energy Standard for Buildings Except Low-Rise Residential Buildings,” 2019.

[3] Canadian Commission on Building and Fire Codes, National Energy Code of Canada for Buildings, 2017. National Research Council Canada, 2017.

[4] I. Dincer and M. Rosen, Thermal Energy Storage Systems and Applications, 2nd ed. John Wiley and Sons, Ltd., 2011.

[5] F. Kazhamiaka, G. F. Yashar, S. Keshav, and C. Rosenberg, "Robust and practical approaches for solar PV and storage sizing," e-Energy 2018 - Proc. 9th ACM Int. Conf. Futur. Energy Syst., pp. 146-156, 2018.

[6] S. Pinnau and C. Breitkopf, "Determination of Thermal Energy Storage (TES) characteristics by Fourier analysis of heat load profiles," Energy Convers. Manag., vol. 101, pp. 343-351, 2015.

[7] M. Bianchi, A. De Pascale, and P. R. Spina, "Guidelines for residential micro-CHP systems design,” Appl. Energy, vol. 97, pp. 673-685, 2012.

[8] R. and A.-C. E. American Society of Heating, "2016 ASHRAE Handbook-HVAC Systems and Equipment," M. S. Owen, Ed. 2016.

[9] K. C. Kavvadias, A. P. Tosios, and Z. B. Maroulis, "Design of a combined heating, cooling and power system: Sizing, operation strategy selection and parametric analysis," Energy Convers. Manag., vol. 51, no. 4, pp. 833-845, 2010.

[10] J. M. Cabello, J. M. Cejudo, M. Luque, F. Ruiz, K. Deb, and R. Tewari, "Optimization of the sizing of a solar thermal electricity plant: Mathematical 
programming versus genetic algorithm," 2009 IEEE Congr. Evol. Comput. CEC 2009, pp. 1193-1200, 2009.

[11] K. Wang, M. A. Satyro, R. Taylor, and P. K. Hopke, "Thermal energy storage tank sizing for biomass boiler heating systems using process dynamic simulation," Energy Build., vol. 175, pp. 199-207, 2018.

[12] M. C. Rodríguez-Hidalgo, P. A. Rodríguez-Aumente, A. Lecuona, M. Legrand, and R. Ventas, "Domestic hot water consumption vs. solar thermal energy storage: The optimum size of the storage tank," Appl. Energy, vol. 97, pp. 897-906, 2012.

[13] T. Agami Reddy, Applied Data Analysis and Modeling for Energy Engineers and Scientists, 1st ed. Springer US, 2011.

[14] T. Bradford, The Energy System: Technology, Economics, Markets and Policy. Cambridge, Massachusetts: The MIT Press, 2018.

[15] P. Pinel, C. A. Cruickshank, I. Beausoleil-Morrison, and A. Wills, "A review of available methods for seasonal storage of solar thermal energy in residential applications," Renew. Sustain. Energy Rev., vol. 15, no. 7, pp. 3341-3359, 2011.

[16] University of Wisconsin-Madison. Solar Energy Laboratory, "Storage Tank TESS Library," vol. 11, pp. 1-40.

[17] J. F. James, A Student's Guide to Fourier Transforms: With Applications in Physics and Engineering. Cambridge University Press, 2012.

[18] J. W. Cooley and J. W. Tukey, "An Algorithm for the Machine Calculation of Complex Fourier Series,” vol. 19, no. 90, pp. 297-301, 1965.

[19] R. Bellman, Adaptive control processes: a guided tour. Princeton, N.J.: Princeton University Press, 1961. 
[20] University of Wisconsin-Madison. Solar Energy Laboratory, "TRNSYS 18 A Transient System Simulation Program - Volume 8 Weather Data," vol. 8. 2017.

[21] J. Coady, K. LaFreniere, J. Russel, J.-P. Chabot, J. Duquette, and S. Schott, “Sustainable Energy Options - For the Future of Mocreebec Eeyoud," 2019.

[22] S. Edwards, I. Beausoleil-Morrison, and A. Laperrière, "Representative hot water draw profiles at high temporal resolution for simulating the performance of solar thermal systems," Sol. Energy, vol. 111, pp. 43-52, 2015.

[23] K. LaFreniere, "Assessing Tradeoffs Between Solar Thermal and Wind Energy Integration in an Isolated Community Electrical-Thermal Grid," MASc thesis. Carleton University, 2020.

[24] S. A. Klein et al, "TRNSYS 18: A Transient Simulation Program." Solar Energy Laboratory, University of Wisconsin, Madison, USA, 2017.

[25] The University of Wisconsin-Madison, "Papers and Validation." [Online]. Available: https://sel.me.wisc.edu/trnsys/validation/index.html. [Accessed: 23May-2020].

[26] American Society of Heating Refrigeration and Air-Conditioning Engineers, "Cooling Towers," in 2016 ASHRAE Handbook - HVAC Systems and Equipment, ASHRAE, 2016.

[27] ASHRAE, "Hydronic heat-distributing units and radiators," in ASHRAE Handbook - Heating, Ventilating, and Air-Conditioning Systems and Equipment, 2016.

[28] M. Jones, "Coupling TRNSYS and MATLAB for Genetic Algorithm Optimization in Sustainable Building Design,” in BauSIM, 2010, pp. 9-14.

[29] J. Kelble and R. S. Means Company, Mechanical Costs with RSMeans Data, 2019, 
42nd ed. Rockland: MA: Gordion, 2018.

[30] D. Fooladivanda, C. Rosenberg, and S. Garg, "An analysis of energy storage and regulation," 2014 IEEE Int. Conf. Smart Grid Commun. SmartGridComm 2014, vol. 7, no. 4, pp. 91-96, 2015.

[31] A. D. Moore, T. Urmee, P. A. Bahri, S. Rezvani, and G. F. Baverstock, "Life cycle assessment of domestic hot water systems in Australia," Renew. Energy, vol. 103, pp. 187-196, 2017.

[32] A. Hobbi and K. Siddiqui, "Optimal design of a forced circulation solar water heating system for a residential unit in cold climate using TRNSYS," Sol. Energy, vol. 83, no. 5, pp. 700-714, 2009.

[33] R. Tuckett, "Short-Term Energy Outlook ( STEO ) Forecast highlights," US EIA Short-Term Energy Outlook, no. April 2017, pp. 1-48, 2018.

[34] GE Energy Consulting, "Pan-Canadian Wind Integration Study (PCWIS) Final Report Prepared for: Canadian Wind Energy Association (CanWEA) Legal Notices," vol. 2016, no. Revision 3, p. 367, 2016.

[35] M. Jiménez-Arreola, R. Pili, F. Dal Magro, C. Wieland, S. Rajoo, and A. Romagnoli, "Thermal power fluctuations in waste heat to power systems: An overview on the challenges and current solutions," Appl. Therm. Eng., vol. 134, no. November 2017, pp. 576-584, 2018.

[36] C. Rockenbaugh et al., "High Performance Flat Plate Solar Thermal Collector Evaluation," 2016.

[37] The Dow Chemical Company, "Technical Data Sheet DOWFROST TM Heat Transfer Fluid." [Online].

Available: 
http://msdssearch.dow.com/PublishedLiteratureDOWCOM/dh_09e3/0901b803809 e3e36.pdf?filepath=/heattrans/pdfs/noreg/180-01314.pdf\&fromPage=GetDoc.

[Accessed: 26-Mar-2020].

[38] Hydro Québec, "Rate D," 2020. [Online]. Available: https://www.hydroquebec.com/residential/customer-space/rates/rate-d.html. [Accessed: 01-Sep-2020].

[39] S. S. Sun, F. Kazhamiaka, S. Keshav, and C. Rosenberg, "Using synthetic traces for robust energy system sizing," e-Energy 2019 - Proc. 10th ACM Int. Conf. Futur. Energy Syst., pp. 251-262, 2019.

[40] F. Kazhamiaka, C. Rosenberg, and S. Keshav, "Practical Strategies for Storage Operation in Energy Systems: Design and Evaluation," IEEE Trans. Sustain. Energy, vol. 7, no. 4, pp. 1602-1610, 2016. 